\title{
Lonicerae Japonicae Flos and Lonicerae Flos: A Systematic Pharmacology Review
}

\author{
Yujie Li, ${ }^{1}$ Weiyan Cai, ${ }^{1}$ Xiaogang Weng, ${ }^{1}$ Qi Li, ${ }^{1}$ Yajie Wang, ${ }^{1}$ Ying Chen, ${ }^{1}$ Wei Zhang, \\ Qing Yang, ${ }^{1}$ Yan Guo, ${ }^{1}$ Xiaoxin Zhu, ${ }^{1}$ and Hainan Wang ${ }^{2}$ \\ ${ }^{1}$ Institute of Chinese Materia Medica, China Academy of Chinese Medical Sciences, No.16, Dongzhimen Nei Nanxiao Road, \\ Dongcheng District, Beijing 100700, China \\ ${ }^{2}$ Department of Drug and Cosmetics Registration, China Food and Drug Administration, Xuanwumen Xidajie, Beijing 100053, China \\ Correspondence should be addressed to Xiaoxin Zhu; xxzhu@icmm.ac.cn and Hainan Wang; md_wanghainan@126.com
}

Received 1 April 2015; Accepted 23 June 2015

Academic Editor: Kuzhuvelil B. Harikumar

Copyright (C) 2015 Yujie Li et al. This is an open access article distributed under the Creative Commons Attribution License, which permits unrestricted use, distribution, and reproduction in any medium, provided the original work is properly cited.

Lonicerae japonicae flos, a widely used traditional Chinese medicine (TCM), has been used for several thousand years in China. Chinese Pharmacopeia once included Lonicerae japonicae flos of Caprifoliaceae family and plants of the same species named Lonicerae flos in general in the same group. Chinese Pharmacopeia (2005 Edition) lists Lonicerae japonicae flos and Lonicerae flos under different categories, although they have the similar history of efficacy. In this study, we research ancient books of TCM, 4 main databases of Chinese academic journals, and MEDLINE/PubMed to verify the origins and effects of Lonicerae japonicae flos and Lonicerae flos in traditional medicine and systematically summarized the research data in light of modern pharmacology and toxicology. Our results show that Lonicerae japonicae flos and Lonicerae flos are similar pharmacologically, but they also differ significantly in certain aspects. A comprehensive systematic review and a standard comparative pharmacological study of Lonicerae japonicae flos and Lonicerae flos as well as other species of Lonicerae flos support their clinical safety and application. Our study provides evidence supporting separate listing of Lonicerae japonicae flos and Lonicerae flos in Chinese Pharmacopeia as well as references for revision of relevant pharmacopeial records dealing with traditional efficacy of Lonicerae japonicae flos and Lonicerae flos.

\section{Introduction}

Lonicerae japonicae flos (also Jinyinhua in Chinese), a plant species in traditional Chinese medicine (TCM), has been widely used as a drug for several millennia with confirmed curative effects. It has been recorded in the Chinese Pharmacopeia (1963 Edition), limiting the therapeutic use of Lonicerae japonicae flos to the dried flower buds of Lonicera japonica Thunb., which belongs to Caprifoliaceae. In the 1977, 1985, 1990, 1995, and 2000 Editions of Chinese Pharmacopeia, three other plant sources were also listed in the category of Lonicerae japonicae flos including the dried flower buds or initial flowers of Lonicera hypoglauca Miq., Lonicera confusa DC., and Lonicera dasystyla Rehd. Chinese Pharmacopeia (2005 Edition) lists Lonicerae japonicae flos and Lonicerae flos as independent items based on differences in medicinal history, plant morphology, medicinal properties, and chemical constituents, and the only plant source of Lonicerae japonicae flos is again limited to Lonicera japonica Thunb. Lonicerae flos has three sources of germplasm including Lonicera macranthoides Hand.-Mazz., Lonicera hypoglauca Miq., and Lonicera confusa DC. Chinese Pharmacopeia (2010 Edition) adds Lonicera fulvotomentosa Hsu et S. C. Cheng to Lonicerae flos following the 2005 Edition, making the aforementioned 4 plant species legal for Lonicerae flos. However, the descriptions of flavor, meridian tropism (MT), functions, and indications are not different between Lonicerae japonicae flos and Lonicerae flos in Chinese Pharmacopeia although they have different sources.

In order to comprehensively review the pharmacological studies, we investigate the efficacy of Lonicerae japonicae flos and Lonicerae flos in ancient books of TCM and 
searched for the literatures both at home and abroad from the China Academic Journal Network Publishing Database of the China National Knowledge Infrastructure, Wanfang Database, China Biomedical Database, and MEDLINE/PubMed. A total of 2864 papers relevant to Lonicerae japonicae flos and Lonicerae flos are retrieved before August 2014, of which 514 dealt with pharmacological effects of main ingredients of Lonicerae japonicae flos and Lonicerae flos. The literatures are further collated to summarize the studies with clear information of the origin of species. Pharmacological advances are also systematically reviewed to provide references for scientific appreciation of Lonicerae japonicae flos and Lonicerae flos.

\section{Traditional Records of Lonicerae Japonicae Flos [1]}

Lonicerae japonicae flos is termed "Rendong" in ancient books of TCM. The Collective Notes to Canon of Materia Medica (around 480-498 AD) writes: "It grows everywhere and is classified into liane. It does not fade over winter and thus be named as Rendong." Since then only the name "Rendong" has been recorded in all the medical books until Tang Dynasty. Subsequently in Song Dynasty, The Prescriptions of Su and Shen (960-1127 AD) and Lvchanyan Materia Medica (1220 AD) used the term "Lonicerae japonicae flos" as a herb also named as Rendong.

Before Ming Dynasty, the Collective Notes to Canon of Materia Medica, Annotation of Materia Medica, and The Prescriptions of Su and Shen only provided a brief description of Rendong and the recorded characteristics of the original plant including liane, opposite leaf, and leaf shape consistent with those of Caprifoliaceae. In Ming Dynasty, the Compendium of Materia Medica (1578 AD) offered the most detailed description of Lonicera flower that "it blossoms in March and April with the length of flower over 3 decimeters. One pedicel contains 2 flowers and each flower has 2 petals either large or narrow with half-edge structure. It has stamens and pistils. The early flower has white stamens, pistils, and petals that turn yellow in 2 to 3 days." In the ancient Materia Medica, An Illustrated Book of Plants authored by Wu Qijun in Qing Dynasty included the ink drawings of Lonicerae japonicae flos with accurate proportion and precise morphology. In addition, the Collected Essentials of Species of Materia Medica written in Ming Dynasty also contains representative colored illustrations of Lonicerae japonicae flos, and the archives show clearly the typical morphological characteristics of Caprifoliaceae including liane, 1 pedicel containing 2 flowers, lip-like and white flowers, opposite leaf, ovate shape, and lobate and ovate shape.

In summary, the original plant morphology of Lonicerae japonicae flos is basically similar in the studies before Qing Dynasty. No records of the medicinal use of Lonicerae flos have been found in ancient books. Based on comparative plant morphology, only Lonicera japonica Thunb., among 21 varieties of Lonicera, is consistent with the morphological characteristics of traditional medicinal Lonicerae japonicae flos while the other 4 species in Chinese Pharmacopeia (2010 Edition) were markedly different in origin.
According to Chinese Pharmacopeia (2005 and 2010 Editions), both Lonicerae japonicae flos and Lonicerae flos are sweet in flavor and cold in nature, attributed to lung, heart, and stomach meridians. They clear heat, toxins, and certain external ailments. They are indicated for carbuncles and pyocutaneous disease, pharyngitis, erysipelas, heat toxins, blood dysentery, exogenous hot ailments, and febrile diseases.

\section{Modern Pharmacological Studies on Lonicerae Japonicae Flos and
Lonicerae Flos}

Lonicerae japonicae flos grows mainly in Shandong, Shaanxi, Henan, and Hebei Provinces in China, among which the Pingyi County, Linyi City, Shandong Province, is the main area with the largest production. Lonicerae flos is mainly grown in the provinces located at the southern regions of Yangtze River such as Hunan Province, Sichuan Province, Guangdong Province, Guizhou Province, and Guangxi Province. Recent studies have been focused on the pharmacological effects of Lonicerae japonicae flos. In contrast, the studies of Lonicerae flos are comparatively limited and most studies focus on Lonicera macranthoides Hand.-Mazz., which exhibits basically similar pharmacological effects as those of Lonicerae japonicae flos.

\subsection{Antibacterial Effects}

3.1.1. Lonicerae Japonicae Flos. Many pharmacologic studies have clearly confirmed the bacteriostatic and antibacterial effects of Lonicerae japonicae flos both in vivo and in vitro. Compared with other commonly seen antibacterial drugs, Lonicerae japonicae flos exhibits a broader antimicrobial spectrum, more powerful antibacterial activity, and inhibition of drug-resistant bacteria. The antibacterial activities of Lonicerae japonicae flos were detailed in Table 1.

Components of Lonicerae japonicae flos including water extract, alcohol extract, polysaccharide, and volatile oil can extensively inhibit Gram-negative bacteria and Gram-positive bacteria including Streptococcus haemolyticus, Staphylococcus aureus, Salmonella Typhi, Klebsiella pneumoniae, Salmonella paratyphi, Vibrio cholerae, oral pathogens, Diplococcus intracellularis, Streptococcus pneumoniae, Mycobacterium tuberculosis, and Pseudomonas aeruginosa. In particular, the water extracts of Lonicerae japonicae flos strongly inhibit Escherichia coli and Staphylococcus aureus but are comparatively weak against Pseudomonas aeruginosa and Shigella flexneri [2-12]. Based on in vivo antimicrobial tests, Lonicerae japonicae flos is fairly inhibitory to Staphylococcus aureus and Diplococcus pneumoniae but minimally active against other pathogens $[13,14]$. A study reports that $10 \%$ Lonicerae japonicae flos extract has antibacterial effect against Escherichia coli and Staphylococcus aureus, which is equivalent to that of penicillin $100 \mu \mathrm{mol} / \mathrm{mL}$ [15], indicating characteristic high antibacterial activity similar to that of antibiotics.

The components of Lonicerae japonicae flos including water-soluble polysaccharides have remarkable inhibitory 


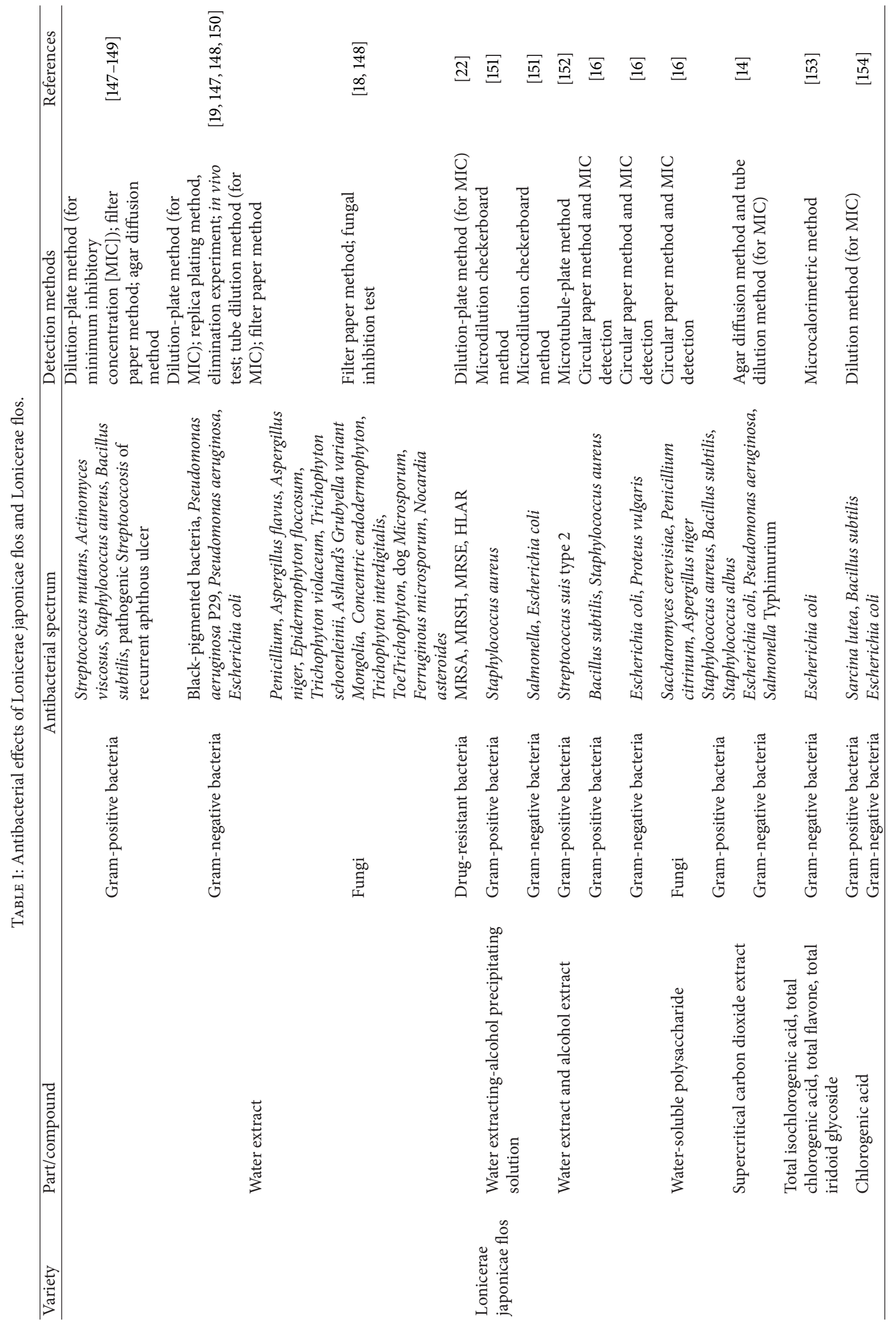




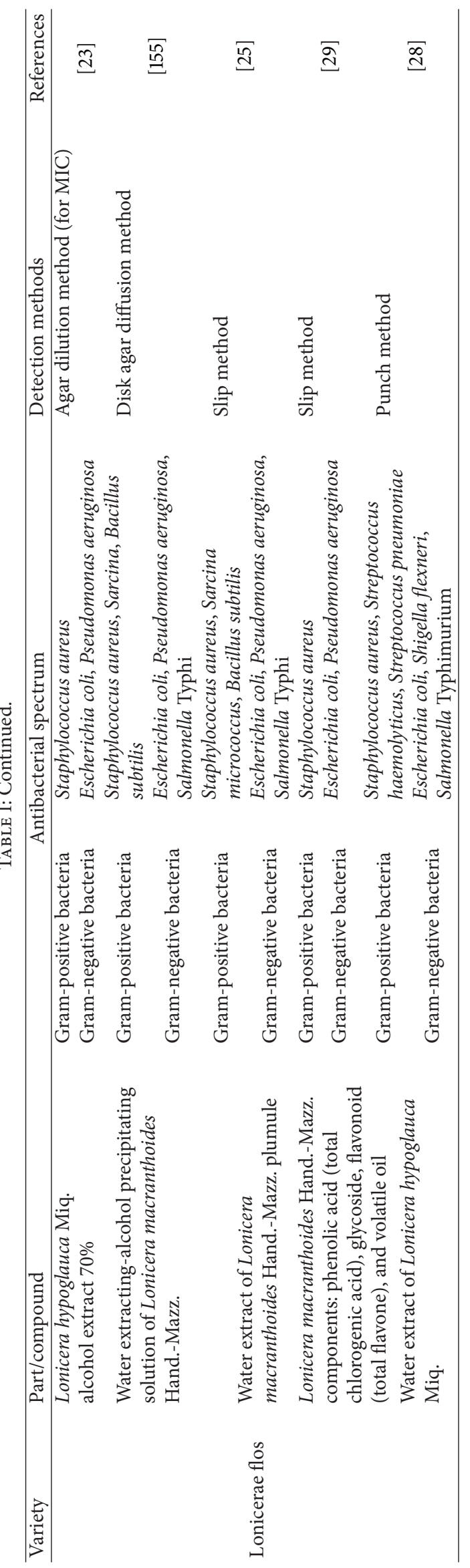


activities against fungi such as dermatophytes, Saccharomyces cerevisiae, Penicillium citrinum, Aspergillus niger, Cryptococcus neoformans, Fusarium moniliforme, Candida albicans, and Aspergillus sp. In addition, the volatile oil of Lonicerae japonicae flos has proven to have antifungal activities [16-18].

Current clinical antimicrobial treatments are associated with a high frequency of multidrug resistance and widespread plasmid resistance. Studies suggest that Lonicerae japonicae flos extracts not only potently inhibit common pathogens but also significantly suppress drug-resistant bacteria. In addition, the water decoction effectively inhibits drug-resistant $\mathrm{R}$ plasmid of Pseudomonas aeruginosa and sensitizes Pseudomonas aeruginosa to single antibiotic. Mice administered with extracts of Lonicerae japonicae flos show improved multiantibiotic sensitivities [19-21]. Lonicerae japonicae flos kills several clinically common drug-resistant bacteria such as methicillin-resistant Staphylococcus aureus (MRSA), methicillin-resistant Staphylococcus haemolyticus (MRSH), methicillin-resistant Staphylococcus epidermidis (MRSE), and high-level aminoglycoside resistant (HLAR) bacteria to varying degrees, and the antibiotic effects are positively correlated with drug concentration [22]. These experimental results further enrich our understanding of the antibacterial activity of Lonicerae japonicae flos and provide scientific data of therapeutic efficacy against drug-resistant pathogens.

3.1.2. Lonicerae Flos. Reports of efficacy and pharmacology of Lonicerae flos suggest several effective antifungal and antibacterial extracts (see Table 1).

The ethanol extracts, water extracting-alcohol precipitating solution, and water extracts of Lonicerae flos are strongly inhibitory against multiple pathogens such as Pseudomonas aeruginosa, Bacillus subtilis, Staphylococcus aureus, Candida albicans, Escherichia coli, Salmonella Typhi, Shigella dysenteriae, and Pseudomonas aeruginosa [23-25] and effectively protected Staphylococcus aureus-infected mice [26].

Lonicerae flos shows various antibacterial activities. The water extracts of Lonicera macranthoides Hand.-Mazz. are the most effective against Staphylococcus aureus and group B Streptococcus and effective against Salmonella Typhi, Escherichia coli, and Shigella dysenteriae but poorly effective against Proteus vulgaris [27]. Lonicerae flos (Lonicera macranthoides Hand.-Mazz., Lonicera hypoglauca Miq.) from Sichuan has inhibitory effects against Staphylococcus aureus, Streptococcus haemolyticus, Escherichia coli, Shigella flexneri, Salmonella Typhimurium, and Streptococcus pneumoniae, but not against Streptococcus haemolyticus and Salmonella Typhimurium [28]. Although reports of antibacterial activity of Lonicerae flos vary, they still clearly indicate that the antibacterial pharmacologic activity of Lonicerae flos is markedly different depending on bacteriostatic effect.

The antibacterial effects of Lonicerae flos extracts vary with the different types and methods of preparation. For example, Lonicera macranthoides Hand.-Mazz. contains phenolic acids (total chlorogenic acids), glycosides, flavonoids (total flavones), and volatile oil that inhibit Escherichia coli, Staphylococcus aureus, and Pseudomonas aeruginosa to different degrees, with greater inhibition of Staphylococcus aureus than Escherichia coli. Further, phenolic acids have significantly better antibacterial effects than others [29].

\subsection{Antiviral Effects}

3.2.1. Lonicerae Japonicae Flos. Lonicerae japonicae flos extracts and its active components including chlorogenic acid, flavonoid, caffeoylquinic acid, and iridoid glycoside can inhibit herpes simplex keratitis [30], influenza virus pneumonia [31], influenza A virus [31-33], porcine reproductive and respiratory syndrome virus [34], Newcastle disease virus [35], respiratory syncytial virus [36-38], influenza virus [39], human cytomegalovirus [40], and so on. In addition, the extracts can significantly inhibit and inactivate cytomegalovirus in guinea pigs [41], pseudorabies virus strain MinA [42], influenza virus variant FM1 [31], Coxsackie $\beta_{3}$ virus $[36,43]$, enteric cytopathic human orphan 19 virus [43], and so forth. The mechanism of action entails enhancing the binding of drug with ceramidase [44] and increases cellular antiviral potency [45] and organ protection in influenza [46].

3.2.2. Lonicerae Flos. The water extracts of Lonicera macranthoides Hand.-Mazz. significantly inhibit the infectivity of 293 cells by defective adenovirus (Ad-lacZ) $[45,46]$. The alcoholsoluble component of Lonicera hypoglauca Miq. named pheophytin is a hepatitis $\mathrm{C}$ virus $(\mathrm{HCV}) \mathrm{NS} 3$ inhibitor $\left(\mathrm{IC}_{50}\right.$ $0.89 \mu \mathrm{M}$ ), which decreases the expression of HCV viral proteins and ribonucleic acids with dose-dependent effect [47].

The flavone extracts of Lonicerae flos (Lonicera macranthoides Hand.-Mazz.) significantly inhibit and inactivate pseudorabies virus (PRV) infection of Vero cells [42]. The active components called chlorogenic acids significantly suppress the Newcastle disease virus (NDV) infection in Vero cells [48] and chlorogenic acids significantly inhibit the proliferation of NDV in Vero cells [49].

\subsection{Anti-Inflammatory Effects}

3.3.1. Lonicerae Japonicae Flos. The water extracts of Lonicerae japonicae flos have significant anti-inflammatory effects on classical inflammatory models such as carrageenan/formaldehyde-induced rat paw swelling [50], mouse ear edema $[13,51]$, cotton ball granulomatous hyperplasia, mouse cutaneous vascular hyperpermeability [52], and egg whiteinduced localized acute inflammation [53]. Furthermore, it also exhibits anti-inflammatory effect in rat cervicitis model [54, 55], Escherichia coli-infected mouse model [56], excision wound model of infected rat [57], and ovalbumin-induced rat asthma model [58]. The mechanisms include inhibition of inflammatory factor synthesis/release, decreased expression of immune related molecules, and enzyme activities of matrix metalloproteinase.

The water extracts of Lonicerae japonicae flos inhibit the production of histamine and the expression of L-histamine decarboxylase by cultured human keratinocytes [59]. Further, it inhibits the production of nitric oxide and secretion of tumor necrosis factor-alpha (TNF- $\alpha$ ) by Raw264.7 cells with dose-dependent effects [60] and prevents the trypsininduced mast cell activation by suppressing the extracellular 
TABLE 2: Anti-inflammatory effects of Lonicerae japonicae flos and Lonicerae flos.

\begin{tabular}{|c|c|c|c|}
\hline Species & Part/compound & Detection methods & References \\
\hline \multirow{7}{*}{$\begin{array}{l}\text { Lonicerae } \\
\text { japonicae flos }\end{array}$} & Water extract & $\begin{array}{l}\text { Carrageenan-induced paw swelling model, } \\
\text { dimethylbenzene/formalin-induced inflammatory } \\
\text { model, cotton ball granulomatous hyperplasia model, } \\
\text { cervicitis model, Escherichia coli infection model, } \\
\text { Raw264.7 cell activation model, trypsin-induced mast } \\
\text { cell activation model, human keratinocyte } \\
\text { three-dimensional culture inflammatory activation } \\
\text { model }\end{array}$ & {$[50,52,54,56,59-61]$} \\
\hline & Alcohol extract $30 \%$ & Croton oil-induced inflammatory model & {$[55]$} \\
\hline & Alcohol extract $57 \%$ & $\begin{array}{l}\text { Egg white-induced paw swelling model, } \\
\text { dimethylbenzene-induced inflammatory model }\end{array}$ & {$[51,53]$} \\
\hline & Alcohol extract 70\% & LPS-induced Raw264.7 cell activation model & {$[62]$} \\
\hline & Alcohol extract 95\% & $\begin{array}{l}\text { Resection wound model, H1N1-infected human } \\
\text { bronchial epithelial cell line A549 model }\end{array}$ & {$[57,156]$} \\
\hline & Alcohol extract & Ovalbumin-induced asthma model & {$[58]$} \\
\hline & Supercritical carbon dioxide extract & Dimethylbenzene-induced inflammatory model & {$[51]$} \\
\hline \multirow{4}{*}{ Lonicerae flos } & $\begin{array}{l}\text { Water extract of Lonicera } \\
\text { macranthoides Hand.-Mazz. }\end{array}$ & $\begin{array}{l}\text { Formalin pain model, acetic acid-writhing model, } \\
\text { xylene-induced inflammation model, } \\
\text { carrageenan-induced paw swelling model, yeast } \\
\text { thermal model }\end{array}$ & {$[85]$} \\
\hline & $\begin{array}{l}\text { Water extract of Lonicera } \\
\text { macranthoides Hand.-Mazz. flower }\end{array}$ & $\begin{array}{l}\text { Acetic acid-induced capillary permeability increase } \\
\text { model, paw swelling model, cotton ball granulomatous } \\
\text { hyperplasia model }\end{array}$ & {$[102]$} \\
\hline & $\begin{array}{l}\text { Supercritical carbon dioxide extract of } \\
\text { Lonicera macranthoides Hand.-Mazz. }\end{array}$ & $\begin{array}{l}\text { Acetic acid-induced abdominal capillary permeability } \\
\text { increase model, carrageenan-induced paw swelling } \\
\text { model, pleuritis model, cotton ball granulomatous } \\
\text { hyperplasia model }\end{array}$ & {$[65,66]$} \\
\hline & $\begin{array}{l}\text { Water extract } 50 \% \text { and alcohol extract } \\
95 \% \text { of Lonicera hypoglauca Miq. }\end{array}$ & $\begin{array}{l}\text { Dimethylbenzene-induced inflammation model, } \\
\text { carrageenan-induced paw swelling model, cotton ball } \\
\text { granulomatous hyperplasia model }\end{array}$ & {$[64]$} \\
\hline
\end{tabular}

signal-regulated kinase (ERK) phosphorylation [61]. The polyphenols of Lonicera japonica Thunb. downregulate proinflammatory mediators and counter the lipopolysaccharidesinduced inflammatory response of Raw264.7 cells by inhibiting NF- $\kappa$ B p 65 nuclear translocation and p38 MAPK phosphorylation [62].

According to the Min Jiang research, it is clear that the anti-inflammatory activity of Flos Lonicerae Japonicae (FLJ) has profound material basis. By using UPLC-Q/TOF-MS and dual luciferase reporter gene assay, they revealed the potent $\mathrm{NF}-\kappa \mathrm{B}$ inhibition influence of the extract from FLJ, which could be classified into 2 types: chlorogenic acid and iridoid glycosides, including swertiamarin. More importantly, as reported in this study, the anti-inflammatory activity decreased during the flowering phases progression. This result suggested that the intensity of anti-inflammatory efficacy of FLJ is dynamically changed in distinct flowering phases, indicating that the effective components temporally affect the clinical application. Taken together, the molecular based quality control (including chlorogenic acid, swertiamarin, and sweroside) and the optimized pharmacological practice are extremely needed and urgent for FLJ [63].

3.3.2. Lonicerae Flos. Water and alcohol extracts of the leaves and flowers of Lonicerae flos have anti-inflammatory effects on dimethylbenzene-induced mouse ear edema, carrageenan-induced rat swelling, and cotton ball granulomatous hyperplasia model $[23,64]$. The volatile organic compounds of Lonicerae flos (Lonicera macranthoides Hand.-Mazz.) have some inhibitory effects on acetic acid-induced abdominal capillary permeability increase in mice, mouse ear edema, rat pleuritis, and cotton ball granulomatous hyperplasia, significantly lower the concentrations of prostaglandin $\mathrm{E}_{2}$ $\left(\mathrm{PGE}_{2}\right)$ and malondialdehyde of the carrageenan-induced paw swelling in norepinephrine animals, and decrease the contents of $\mathrm{PGE}_{2}$ and $\mathrm{NO}$ in acute pleural effusion of rat $[23,65,66]$. Moreover, the water extracts of Lonicerae flos (Lonicera macranthoides Hand.-Mazz.) have significant antiinflammatory effects by inhibiting the increased capillary permeability in mice, rat paw swelling, and cotton ball granulomatous hyperplasia [25]. The anti-inflammatory effects of both Lonicerae japonicae and Lonicerae flos were listed in Table 2.

\subsection{Antioxidative Effects}

3.4.1. Lonicerae Japonicae Flos. The major antioxidants in Lonicerae japonicae flos include neochlorogenic acid, chlorogenic acid, 4-dicaffeoylquinic acid, caffeic acid, isochlorogenic acid $\mathrm{A}$, isochlorogenic acid $\mathrm{B}$, isochlorogenic acid $\mathrm{C}$, rutin, xylostein, isoquercitrin, luteolin-7-O-glucoside, and luteolin $[67,68]$.

The alcohol extracts of Lonicerae japonicae flos have antioxidative effects on edible rapeseed oil, peanut oil, ghee, 
salad oil, mutton tallow [69], linoleic acid, and lard [70]. The crude extracts of chlorogenic acid and total flavones have an inhibitory effect on the antioxidative reaction of oil and prevent the autoxidation of linoleic acid and lard. Further, their redox capacities are 2.0- and 2.8-fold higher than that of synthetic antioxidant butylated hydroxyanisole (BHA) [71, 72]. The antioxidative effects of water and alcohol extracts of Lonicerae japonicae flos on oil may be closely related to the clearance of 2,2-diphenyl-1-picrylhydrazyl (DPPH) and the inhibition of oxygen free radical chain reaction in oil $[73,74]$.

The ultrasonic-treated extracts and decoction of Lonicerae japonicae flos can both scavenge hydrogen peroxide $\left(\mathrm{H}_{2} \mathrm{O}_{2}\right)$, hydroxyl radical $(\mathrm{OH})$, and superoxide radical $\left(\mathrm{O}^{-2}\right)$ [75]. The reducing power and the clearance of $\mathrm{OH}$ are positively correlated with the chlorogenic acid content of Lonicerae japonicae flos. Though the alcohol extracts and methanol extracts have better reducing power and higher clearance rates of ${ }^{\prime} \mathrm{OH}$ than water extracts, the water extracts still have a higher clearance rate of DPPH radicals and a stronger chelating ability with $\mathrm{Fe}^{2+}$ [76]. In addition, the direct clearance of $\mathrm{H}_{2} \mathrm{O}_{2}$ by water extracts effectively reduces tissue injuries in scalded mice [77]. Analysis of antioxidative effects in vitro reveals that the fermented and alcohol extracts of Lonicerae japonicae flos inhibit tyrosinase in mushroom $\left(\mathrm{ED}_{50} 4.07 \mathrm{mg} / \mathrm{mL}\right.$ and $6.93 \mathrm{mg} / \mathrm{mL}$, resp.). Compared with alcohol extracts, fermented extracts are more effective in promoting the clearance of DPPH $\left(\mathrm{ED}_{50} 0.207 \mathrm{mg} / \mathrm{mL}\right)$ and superoxide [78].

Lonicerae japonicae flos significantly upregulates the antioxidant enzyme system of human liver rat basophilic leukemia cell and downregulates NF- $\kappa \mathrm{B}$ signal transduction pathway $[79,80]$. Moreover, it significantly increases the antioxidant enzyme activities of D-galactose-induced aging model in mice, inhibits lipid peroxidation of liver and kidney tissues, and reduces the oxidative damage in human body [81].

\subsection{Antipyretic Effects}

3.5.1. Lonicerae Japonicae Flos. Lonicerae japonicae flos has antipyretic effects in dry yeast-induced rat fever model [82] and the IL-1 $\beta$-induced fever model in New Zealand rabbits [83], possibly due to the expression of prostaglandin E2 receptor EP3 at the preoptic area of hypothalamus (POAH) neurons [82]. Further, it reduces injuries caused by free radicals and improves human immunity [84].

3.5.2. Lonicerae Flos. The water extracts and alcohol extracts of both Lonicera macranthoides Hand.-Mazz. and Lonicera hypoglauca Miq. effectively neutralize yeast-induced hyperthermia in rats $[64,85,86]$.

\subsection{Liver Protection}

3.6.1. Lonicerae Japonicae Flos. The water extracts of Lonicerae japonicae flos containing $20 \%$ chlorogenic acid are protective against alcohol-induced chemical liver injury in mice [87], also it is same liver protection for the water extracts of Lonicerae japonicae flos to the mouse model of acute liver injury induced by intraperitoneal injection of carbon tetrachloride $\left(\mathrm{CCl}_{4}\right)[88]$.

3.6.2. Lonicerae Flos. Two species of Lonicerae flos (Lonicera fulvotomentosa Hsu et S. C. Cheng and Lonicera macranthoides Hand.-Mazz.) confer different degrees of protection against rat/mouse liver injuries induced by $\mathrm{CCl}_{4}$, D-galactosamine (D-Gal), and acetaminophen (AAP) [89]. Saponins of Lonicera fulvotomentosa Hsu et S. C. Cheng resolve AAPinduced liver injuries by lowering the cytochrome P450 concentration in liver cells of mouse [90-92]. The total saponins of Lonicera fulvotomentosa Hsu et S. C. Cheng significantly alleviate $\mathrm{CCl}_{4}$-induced liver injuries, reduce liver injuries of the patient, and effectively lower the incidence of liver necrotizing changes and the total amount of spotty necrosis. Chlorogenic acid has potent choleretic action that not only significantly increases bile secretion volume but also alleviates chromium-induced liver injuries $[93,94]$.

\subsection{Immunoregulation}

3.7.1. Lonicerae Japonicae Flos. Lonicerae japonicae flos decoction effectively improves human immunity, increases macrophage count, elevates phagocytic ratio and lymphocyte transformation rate [82], and enhances the secretion function of Th1 cells [95]. Lonicerae japonicae flos also promotes the phagocytic function of leucocytes. It decreases T-cell $\alpha$ naphthyl acetate percentage of guinea pig and in vitro secretion of neutrophils and remarkably increases the production of IL-2 [96]. The water extracting-alcohol precipitating solution or the flavones of Lonicerae japonicae flos significantly elevate the organ index of immunosuppressed mice [97]. Lonicerae japonicae flos polysaccharides improve mouse splenocyte proliferation [98], markedly enhance immunity, and resolve delayed-type hypersensitivity. Serum hemolysin test shows that Lonicerae japonicae flos polysaccharides enhance humoral immune activities and raise the organ index of immunocompromised animal models, correlated with dosage [99]. Moreover, the water extracts of Lonicerae japonicae flos have significantly regulated immune response in scald-induced immunosuppressive model [100].

The water extracts of Lonicerae japonicae flos effectively substitute the highly toxic immunosuppressants such as cyclosporin A for the induction of immune tolerance. Lonicerae japonicae flos extracts combined with Con A significantly reduce the active degree of T lymphocytes [101] and avoided acute immunological rejection, hence effective in treating graft rejection.

3.7.2. Lonicerae Flos. The water extracting-alcohol precipitating solution of Lonicera macranthoides Hand.-Mazz. significantly enhances thymus index, spleen index, carbon clearance, and macrophage phagocytic index in cyclophosphamide-induced immunocompromised mice and improves the proliferation of abdominal macrophages and splenic lymphocytes, with remarkable immunoregulatory effects $[91,102]$. 


\subsection{Antitumor Effects}

3.8.1. Lonicerae Japonicae Flos. Intraperitoneal injection of Lonicerae japonicae flos polysaccharides $30 \mathrm{mg} / \mathrm{kg}$ and $90 \mathrm{mg} / \mathrm{kg}$ inhibited $23.95 \%$ and $30.02 \%$ of sarcoma S180, respectively, upregulated the expression level of Bax protein in mouse sarcoma S180, and increased Bax/Bcl-2 ratio and serum TNF- $\alpha$ concentration of tumor-bearing mice, indicating an antitumor effect that does not affect the normal growth and immune functions of tumor-bearing mice [103]. The polyphenolic extracts of Lonicerae japonicae flos inhibit proliferation of human hepatoma HepG2 cell line in a dose-dependent manner, decrease the expression of CDK1, CDC25C, cyclin B1, procaspases 3 and 8, and PARP, and promote the phosphorylation of ERK1/2, JNK, and MAPKs and the dephosphorylation of Akt, resulting in G2/M arrest and apoptosis [104].

3.8.2. Lonicerae Flos. The prosapogenin B of Lonicera macranthoides Hand.-Mazz. has relatively strong growth inhibition against several types of tumor cells especially HL-60, with a dose of $1.25-5 \mathrm{mg} / \mathrm{kg}$ corresponding to a $27.41-54.57 \%$ tumor inhibition rate in Lewis tumors. Genechip detection reveals that Lonicera macranthoides Hand.-Mazz. induces differential expression of 20 out of 84 tumor-associated genes in HL-60 cells, mainly via cell-cycle arrest and inhibition of cellular invasion and metastasis $[105,106]$. The macranthoide B of Lonicera macranthoides Hand.-Mazz. inhibit the growth of 6 types of tumor cells, especially HL-60 cells with an $\mathrm{IC}_{50}$ of $3.8 \mu \mathrm{M}$ by activating apoptosis [107]. Studies using highthroughput screening models indicate that the ethyl acetate extracts of Lonicera macranthoides Hand.-Mazz. suppress epidermal growth factor receptor (EGFR) kinase, with $\mathrm{IC}_{50}$ of $2.027 \mu \mathrm{g} / \mathrm{mL}$. In addition, the phenolic acids and flavones in the extract may play a major role in inhibiting EGFR kinase [108].

3.9. Effects on Glucose and Lipid Metabolism. The water extracts of Lonicerae japonicae flos inhibit the alloxan-induced blood glucose elevation in mice [109]. The results of iodinestarch colorimetry and p-nitrophenyl $\alpha$-D-glucopyranoside (PNPG) assay show that the water extracts dose dependently inhibit activities of $\alpha$-amylase and $\alpha$-glycosidase [110]. Another study indicates that Lonicerae japonicae flos extracts lower the triglyceride levels in serum and liver tissues of hyperlipidemia animal model without significantly affecting serum cholesterol, low-density lipoprotein, high-density lipoprotein, and liver tissue cholesterol [111].

3.10. Antiatherosclerotic (As) Effects. The intragastric administration of Lonicera macranthoides Hand.-Mazz. decreases the area of atherosclerotic plaque and plaque-to-wall area ratio and alleviates atherosclerotic changes in apolipoprotein $\mathrm{E}$ (ApoE) gene knockout mice and reduces lipid droplets and cholesterol concentrations of lipid-loaded THP1 macrophages [112].

\subsection{Antiallergic Effects}

3.11.1. Lonicerae Japonicae Flos. The caudal vein of egg white lysozyme-sensitized mice with increased blood supply is reduced by $35 \%$ alcohol extracts of Lonicerae japonicae flos. This phenomenon indicates that $35 \%$ alcohol extracts have antiallergic effects, and the effective components include chlorogenic acid, iridoid, loganin, and sweroside [113].

3.11.2. Lonicerae Flos. The water extracts of Lonicera fulvotomentosa Hsu et S. C. Cheng are used to treat the bowel inflammation and ovalbumin- (OVA-) mediated type I hypersensitivity in mice. It also decreases the serum OVAspecific Ig E level of sensitized mice, relieves focal necrosis and abscission of small intestinal villi epithelial cells in mice, reduces the $\mathrm{IgA}^{+}$plasma cell count of small intestinal lamina propria (LP), smIgA ${ }^{+}$lymphocyte count of Peyer's patches (PP), and mRNA expression of IL- 4 of small intestinal LP and PP, increases TGF- $\beta$ mRNA of intestinal mucosa, and decreases the mRNA expression of small intestinal TNF- $\alpha$ with dose-dependent effects $[114,115]$. Subcutaneous injection of Lonicerae flos total saponins alleviates diarrhea in mice to varying degrees, decreases mast cell aggregation and degranulation, lowers OVA-specific Ig E level, relieves OVAmediated footpad edema, and resolves small intestinal villi inflammation, indicating that total saponins attenuate mouse Ig E- and immunocyte-mediated hypersensitive response $[116,117]$. The volatile organic compounds of Lonicera macranthoides Hand.-Mazz. also inhibit heterologous passive cutaneous anaphylaxis of ear and dextran-induced pruritus in mice [118].

3.12. Antipregnancy Effects. Intraperitoneal injection of alcohol extracts of Lonicerae japonicae flos has inhibited early pregnancy in mice. Intravenous drip also shows good antipregnancy effects in dogs as early as days 20-22 at a dosedependent manner [119].

3.13. Modulating Gut Microbiota. The water extracts of Lonicera fulvotomentosa Hsu et S. C. Cheng significantly improve intestinal folate deficiency and reduce Gram-negative bacterial resistance and intestinal flora imbalance in rat obstructive jaundice model [120]. The water extracts also promote the growth of bifidobacteria and lactobacilli in vitro, but they inhibit the growth at high concentration [121].

3.14. Antiultraviolet Radiation. Administration of water extracting-alcohol precipitating solution of Lonicerae japonicae flos reduces the breakage of wavy elastic fibers in the skin and the coiling degree of mouse model with ultraviolet radiationaged skin injuries, possibly due to the antioxidant effects [122].

3.15. Antiendotoxin Effects. The water extracts $10 \mathrm{mg} / \mathrm{mL}$ of Lonicerae japonicae flos and chlorogenic acids $1 \mathrm{mg} / \mathrm{mL}$ destroy the ultrastructure of endotoxin [123]. 
3.16. Spasmolytic Effects. The decoction of Lonicerae japonicae flos inhibits the motility of isolated small intestine from rabbit and significantly reduces rabbit small intestinal smooth muscle contraction, electrical activity range $\left(\mathrm{IC}_{50}\right.$, $6.30 \mathrm{mg} / \mathrm{mL}$ ), frequency, and area under curve in dosedependent manner. Propranolol, L-NAME, and glibenclamide partly block the inhibitory effects of Lonicerae japonicae flos on rabbit small intestinal smooth muscle contraction [124]. The water extracting-alcohol precipitating solution of Lonicerae japonicae flos also inhibits the motility of rabbit isolated small intestine [125]. The decoction of Lonicerae japonicae flos significantly inhibits acetylcholine-induced intra- and extracellular calcium-mediated smooth muscle contraction [124].

3.17. Antiplatelet Effect. The water extracts of Lonicerae japonicae flos inhibited ADP-induced platelet aggregation with $\mathrm{IC}_{50}$ of $0.028 \mathrm{~g} / \mathrm{L}$. The $\mathrm{IC}_{50}$ of the organic acids in the water extracts are as follows: isomers of chlorogenic acid (4-caffeoylquinic acid, $0.0286 \mathrm{~g} / \mathrm{L}$; 5-caffeoylquinic acid, $1.707 \mathrm{~g} / \mathrm{L})$, caffeic acid $(2.411 \mathrm{~g} / \mathrm{L})$, and isochlorogenic acids (3,4-dicaffeoylquinic acid, $0.026 \mathrm{~g} / \mathrm{L} ; 3,5$-dicaffeoylquinic acid, $0.328 \mathrm{~g} / \mathrm{L}$; and 4,5 -dicaffeoylquinic acid, $0.539 \mathrm{~g} / \mathrm{L}$ ), indicating relatively strong antiplatelet aggregation effects [126]. Extracts from Lonicerae japonicae flos, methyl caffeate (a polyphenol), methyl chlorogenic acid, digicitrin, and so on significantly inhibited superoxide-induced platelet activation and cellular injuries, ADP-induced platelet aggregation, and calcium ionophore A23187-triggered thromboxane synthesis in platelet microparticles [127]. Methyl caffeate, 3,4-di-Ocaffeoylquinic acid, and methyl 3,4-di-O-caffeoylquinate have relatively strong inhibitory effect on platelet aggregation [128].

3.18. Neuroprotective Effect. The water extracts of Lonicerae japonicae flos have potential antiparkinsonian activities and reduce 6-hydroxydopamine-induced SH-SY5Y cytotoxicity. The protective mechanism is closely related to the inhibition of cellular apoptosis and oxidative stress and the activation of MAPKs, PI3K/Akt, and NF- $\kappa$ B pathways [129]. Further, the water extracts neutralize the $\mathrm{H}_{2} \mathrm{O}_{2}$-induced SH-SY5Y neuroblastoma cytotoxicity, apoptosis, and ROS production to protect the nerves, possibly via inhibition of Akt, JNK, p38 MAPK, and ERK1/2 phosphorylation [130]. The fluorescence spectrum analysis using thioflavin-T fluorometric assay and atomic force microscopy reveals that the dextran derived from Lonicerae japonicae flos inhibits $A \beta_{42}$ deposition with dose-dependent effects and reduces the neurotoxicity of $A \beta_{42}$ for SH-SY5Y cells, suggesting potential therapeutic value for Alzheimer's disease [131].

3.19. Toxicity and Adverse Effects. Intragastric administration of aqueous extracts of Lonicerae japonicae flos is not significantly toxic and does not affect the respiration, blood pressure, or urine volume in experimental animals. The $\mathrm{LD}_{50}$ of subcutaneous injection of Lonicerae japonicae flos concrete is $53 \mathrm{~g} / \mathrm{kg}$ for mice [132]. The $\mathrm{LD}_{50}$ of oral administration of Lonicerae japonicae flos is larger than $15 \mathrm{~g} / \mathrm{kg}$ and thus innocuous. The micronucleus test on bone marrow polychromatic erythrocytes of mice and Ames Salmonellal microsome mutagenicity assay do not reveal any mutagenicity of Lonicerae japonicae flos. Sperm shape abnormality tests in mice reveal no genotoxicity of Lonicerae japonicae flos for germ cells of male animals. The anti-early pregnancy assay of SD rat (oral administration) indicates no adverse effects of Lonicerae japonicae flos on the reproductive function of female animals during pregnancy [133].

After intragastric administration of Lonicera macranthoides Hand.-Mazz. decoction, the mice show significantly decreased spontaneous activities and some degree of sleepiness and prone position, which were restored in 24 hours. Most death occurred in 24 hours, before any convulsions or seizure. However, subsequent anatomical observation indicates no lesions in major organs and the $\mathrm{LD}_{50}$ was 73.95 $(69.80-78.34) \mathrm{g} / \mathrm{kg}$ [134]. The maximum dosage of Lonicera macranthoides Hand.-Mazz. buds for mice is $15 \mathrm{~g} / \mathrm{kg}$, and subacute toxicity test shows no dose-related differences in weight, hematology, blood biochemistry, or organ index with normal control group [135].

\section{Comparative Pharmacology and Toxicology of Lonicerae Japonicae Flos and Lonicerae Flos}

According to pharmacopoeia, Lonicerae japonicae flos and Lonicerae flos have similar efficacy, but researchers still compare the pharmacology and toxicity experimentally since the drugs have different origin and geographic distribution.

\subsection{Comparison of Antibacterial and Bacteriostatic Effects.} Lonicera macranthoides Hand.-Mazz. and Lonicerae japonicae flos have similar antibacterial spectra that significantly inhibit and kill Staphylococcus aureus, Escherichia coli, Salmonella Typhi, Shigella dysenteriae, Proteus vulgaris, Streptococcus group B, Sarcina, and Bacillus subtilis, but their effects are still different. The former has more significantly inhibitory effects on Streptococcus group B [27], Escherichia coli, Staphylococcus aureus, and Pneumococcus [136] than Lonicerae japonicae flos. Only Lonicera macranthoides Hand.-Mazz. has germicidal effects on Shigella dysenteriae [137]. Compared with Lonicerae japonicae flos, it has better bactericidal effects on Shigella dysenteriae [44, 137], Sarcina, and Bacillus subtilis [44] and poorer effects on Salmonella Typhi [44] and Escherichia coli [138] while having similar effects on Pseudomonas aeruginosa [138]. Lonicera confusa DC. exhibits inhibitory effect against Staphylococcus aureus and Salmonella Typhi, equivalent to that of Lonicerae japonicae flos, but better than that of Lonicera hypoglauca Miq. Moreover, it has more significant inhibitory effects on Streptococcus haemolyticus than Lonicerae japonicae flos and Lonicera hypoglauca Miq. [139]. Lonicera macranthoides Hand.-Mazz. decoction $40 \mathrm{~g} / \mathrm{kg}$ is protective in Staphylococcus aureus-infected mice, with a significantly longer survival time than Lonicerae japonicae flos [140]. In conclusion, all species of Lonicerae flos are more antibacterial and bacteriostatic. 
4.2. Comparison of Antiviral Effects. The flavone extracts of Lonicera macranthoides Hand.-Mazz. and Lonicerae japonicae flos are significantly inhibitory against pseudorabies virus- (PRV-) infected Vero cells, with Lonicera macranthoides Hand.-Mazz. showing a stronger effect than Lonicerae japonicae flos, but not significantly different in blocking effects [42]. The chlorogenic acid extracts of Lonicera macranthoides Hand.-Mazz. and Lonicerae japonicae flos inhibit Newcastle disease virus that affects Vero cells without any notable differences in efficacy [48]. Lonicera macranthoides Hand.-Mazz. and the alcohol extracts of Lonicerae japonicae flos remarkably enhance the antiadenoviral ability of cells in vitro, not significantly different [45].

4.3. Comparison of Antioxidant Effects. Orthophenanthroline- $\mathrm{Fe}^{2+}$ colorimetry is used to compare the scavenging capacity of $\mathrm{OH}$ in vitro between the water extracts of Lonicera macranthoides Hand.-Mazz. and Lonicerae japonicae flos, which reveals equally strong scavenging capacity of ${ }^{\circ} \mathrm{OH}$ ( $\mathrm{IC}_{50}$ of scavenging rate, Lonicera macranthoides Hand.Mazz. versus Lonicerae japonicae flos, $6.01 \mathrm{mg} / \mathrm{mL}$ versus $10.22 \mathrm{mg} / \mathrm{mL}$ ) [136]. Biochemiluminescence indicates that the $\mathrm{IC}_{50}$ of luminescence inhibition rates of $\mathrm{O}^{-2}$ and $\mathrm{OH}$ were $10.26 \mathrm{mg} / \mathrm{mL}$ and $3.26 \mathrm{mg} / \mathrm{mL}$ for the water extracts of Lonicera macranthoides Hand.-Mazz. and $16.48 \mathrm{mg} / \mathrm{mL}$ and $10.79 \mathrm{mg} / \mathrm{mL}$ for Lonicerae japonicae flos [141]. The total flavones of Lonicera hypoglauca Miq. inhibit lipid oxidation (according to peroxide value test). The detection results of $\mathrm{H}_{2} \mathrm{O}_{2}$-CTMAB-luminol fluorescence system show an $\mathrm{IC}_{50}$ of fluorescence inhibition rate of $2.21 \times 10^{-2} \mathrm{mg} / \mathrm{mL}$ for the total flavones of Lonicera hypoglauca Miq. and $1.54 \times$ $10^{-2} \mathrm{mg} / \mathrm{mL}$ for Lonicerae japonicae flos, indicating different $\mathrm{H}_{2} \mathrm{O}_{2}$ scavenging capacities [142].

4.4. Comparison of Anti-Inflammatory Effects. Lonicera macranthoides Hand.-Mazz. and Lonicerae japonicae flos used at 1 and $10 \mathrm{~g} / \mathrm{kg}$ significantly inhibit the abdominal capillary permeability in mice and suppress carrageenan-induced paw swelling, with no significant differences. Lonicerae japonicae flos $10 \mathrm{~g} / \mathrm{kg}$ inhibits dimethylbenzene-induced ear edema in mice but Lonicera macranthoides Hand.-Mazz. only shows inhibitory trends and the comparison between them reveals significant difference $[44,138]$.

4.5. Comparison of Antipyretic Effects. Lonicera macranthoides Hand.-Mazz. and Lonicerae japonicae flos $20 \mathrm{~g} / \mathrm{kg}$ equally inhibit the fever induced by subcutaneous injection of Saccharomyces cerevisiae in mice, although the latter is effective for a longer time [140]. Lonicera macranthoides Hand.Mazz., $10 \mathrm{~g} / \mathrm{kg}$, shows inhibitory trend against yeast powderinduced fever in mice and the antipyretic effect is slightly weaker than Lonicerae japonicae flos at the same dose [138, 140].

4.6. Comparison of Immunoregulatory Effects. Lonicera macranthoides Hand.-Mazz. and the water extracting-alcohol precipitating concentrated concretes of Lonicerae japonicae flos (main components: total flavones and chlorogenic acid)
$1 \mathrm{~g} / \mathrm{kg}$ and $10 \mathrm{~g} / \mathrm{kg}$ significantly increase spleen index, thymus index, carbon clearance index, and phagocytic index in mice, but not significantly differently $[44,138]$. Lonicerae japonicae flos $10 \mathrm{~g} / \mathrm{kg}$ significantly elevates the white cell count in normal and immunocompromised mice unlike Lonicera macranthoides Hand.-Mazz. [138].

The water extracts of Lonicera fulvotomentosa Hsu et S. C. Cheng alleviate small intestinal villi inflammation in sensitized mice, reduce mast cell aggregation and cell degranulation, increase the whole-mast cell ratio in LP, decrease intestinal histamine release of sensitized mice, lower the levels of IL-4 and OVA-specific Ig E levels in sensitized mice, and resolved OVA-mediated delayed-type hypersensitivity of footpad in mice [143].

4.7. Comparison of Hemostatic Effects. Both Lonicerae japonicae flos and Lonicerae flos (Lonicera confusa DC. and Lonicera hypoglauca Miq.) shorten bleeding time in mice. Lonicerae japonicae flos has similar hemostatic effect equivalent to Lonicera confusa DC. Lonicerae japonicae flos and Lonicera confusa DC. are significantly more hemostatic than Lonicera hypoglauca Miq. [139].

4.8. Comparison of Toxicity and Adverse Effects. According to a comparative study, the $\mathrm{LD}_{50}$ values of Lonicera macranthoides Hand.-Mazz. and Lonicerae japonicae flos are $84.14 \mathrm{~g} /$ $\mathrm{kg}$ and $72.95 \mathrm{~g} / \mathrm{kg}$, respectively, with no significant difference [27].

The water extracts of sun-cured Lonicera macranthoides Hand.-Mazz. are hemolytic. However, the water extracts of steaming sun-cured Lonicera macranthoides Hand.-Mazz. and Lonicerae japonicae flos show hemolytic reaction only after 3 hours [144]. Total saponins of Lonicera fulvotomentosa Hsu et S. C. Cheng also result in mild hemolysis [139].

In active systemic anaphylaxis test using crude water extracting-alcohol precipitating solution of Lonicera macranthoides Hand.-Mazz., four out of six sensitized guinea pigs show dyspnea, gait instability, and Cheyne-Stokes respiration and die in $40 \mathrm{~s}$ to $5 \mathrm{~min}$ after excitation by intravenous injection of chlorogenic acid $15 \mathrm{mg} / \mathrm{kg}$. Two out of six guinea pigs show agitation, rapid respiration, and gait instability in $30 \mathrm{~s}$. In 10 to $15 \mathrm{~min}$, these symptoms disappear and the mice recover. The allergic reaction intensity is graded as strongly positive. After intravenous injection of crude extracts $15 \mathrm{mg} / \mathrm{kg}$, the nonsensitized guinea pigs manifest agitation, gait instability, rapid respiration, spasm, urination, and defecation in $10 \mathrm{~s}$ to $2.5 \mathrm{~min}$ and recover in $20-30 \mathrm{~min}$. The strength of anaphylactic reaction is strongly positive [145].

Some researchers have compared the hypersensitive and anaphylactic reactions of Lonicerae japonicae flos and Lonicerae flos. The intraperitoneal injection of the water extracts and water extracting-alcohol precipitating solution of Lonicerae japonicae flos or Lonicera macranthoides Hand.Mazz. on alternate days for 3 times may lead to hypersensitive reaction in guinea pigs, with possible death. A further study reveals that the degranulation rate (based on $\beta$-hexosaminidase release assay) of rat basophilic leukemia 
cells $(\mathrm{RBL}-2 \mathrm{H})$ induced by the water extracting-alcohol precipitating solution of Lonicera macranthoides Hand.-Mazz. is significantly higher than that of Lonicerae japonicae flos $(11.33 \% \pm 0.78$ versus $8.52 \% \pm 0.44)$, but with similar activation and proliferation inhibition on peripheral blood mononuclear cells [146].

Therefore, compared with Lonicerae japonicae flos, Lonicerae flos may be potentially dangerous.

\section{Summary}

Chinese Pharmacopeia (1963 Edition) records Lonicera japonica Thunb. of Caprifoliaceae family as the only plant source of medicinal Lonicerae japonicae flos, after which a total of 5 editions of pharmacopeias included the origin of Lonicerae japonicae flos and 4 plants of the same genus under the legal species of Lonicerae japonicae flos. In this systematic review, we confirm that the medicinal value of Lonicerae japonicae flos is limited to Lonicera japonica Thunb. of Caprifoliaceae family in traditional TCM books without any historical evidence supporting the medicinal use of Lonicerae flos-related species, thus providing a scientific basis for the independent listing of Lonicerae japonicae flos and Lonicerae flos since Chinese Pharmacopeia (2005 Edition).

The functions and indications of Lonicerae japonicae flos and Lonicerae flos are similar in Chinese Pharmacopeia 2005 and 2010 Editions. Our results confirm similar pharmacological activities of Lonicerae japonicae flos and Lonicerae flos, but the former is more widely studied pharmacologically. Lonicerae japonicae flos has glucose-lowering, antiearly pregnancy, antiultraviolet radiation, antiendotoxin, antiulcer, antiplatelet aggregation, antifertility, and neuroprotective activities that are not reported in Lonicerae flos. Lonicera macranthoides Hand.-Mazz. and Lonicerae japonicae flos have similar antibacterial, antiviral, antiinflammatory, antioxidative, antifebrile, hepatoprotective, immunoregulatory, and antitumor activities. However, the pharmacological effects involving balancing intestinal flora and antiatherosclerotic effects have not been reported in Lonicerae japonicae flos. Antioxidation is the common pharmacological activity of Lonicera hypoglauca Miq., Lonicera confusa DC., and Lonicera fulvotomentosa Hsu et S. C. Cheng. In addition, Lonicera hypoglauca Miq. also has antibacterial, anti-inflammatory, and antipyretic effects; Lonicera confusa DC. has antibacterial and hemostatic effects; Lonicera fulvotomentosa $\mathrm{Hsu}$ et S. C. Cheng has hepatoprotective and antiallergic effects and saponins have mild hemolytic effect.

Further analysis reveals twenty plus studies comparing the pharmacological activities between Lonicerae japonicae flos and Lonicerae flos. Lonicerae flos has certain advantages in terms of antibacterial and other effects. Some studies report better antibacterial and bacteriostatic activities with Lonicera macranthoides Hand.-Mazz. and Lonicera confusa DC. than Lonicerae japonicae flos and more antioxidant activities of Lonicera macranthoides Hand.-Mazz. than Lonicerae japonicae flos. Lonicera macranthoides Hand.-Mazz. and Lonicerae japonicae flos do not differ significantly in antiviral effects. Lonicera confusa DC. and Lonicerae japonicae flos do not differ significantly in hemostatic effect but both are better than Lonicera hypoglauca Miq. Lonicerae japonicae flos is slightly better than Lonicera macranthoides Hand.-Mazz. in anti-inflammatory, antipyretic, and immunoregulatory effects, but no significant difference in toxicity. Some studies have reported adverse events such as hypersensitive/anaphylactic and hemolytic reaction in Lonicera macranthoides Hand.-Mazz. and Lonicera fulvotomentosa $\mathrm{Hsu}$ et S. C. Cheng. Therefore, meticulous screening and identification of the different species are essential to avoid the risk of adverse and toxic effects during the production and clinical use.

In conclusion, modern pharmacological effects of Lonicerae japonicae flos and Lonicerae flos are similar, although a few significant differences should not be neglected. Since a systematic and standard comparative study has not been developed so far, it is difficult to scientifically evaluate their advantages/disadvantages and differences/similarities. We suggest a comprehensive systematic review and a parallel, crossover study to delineate the mechanisms underlying the comparative pharmacological activities of Lonicerae japonicae flos and Lonicerae flos and different species of Lonicerae flos. A comparative analysis of the clinical efficacy and safety of pharmacologically active ingredients/products in Lonicerae japonicae flos and Lonicerae flos is essential to fully and accurately evaluate their effects and toxic side effects. References for the revision of relevant pharmacopoeial records should be provided along with supporting clinical efficacy and safety data.

\section{Conflict of Interests}

The authors have no conflicting financial interests.

\section{Acknowledgments}

This work is supported by grants from the National Natural Science Foundation of China (30973901), International Science and Technology Cooperation Program of China (2011DFA30870), and Joint Research Project of The Twentieth Session of China-Thailand Joint Committee on Science and Technology Cooperation (20-602J). The funders had no role in study design, data collection and analysis, decision to publish, or preparation of the paper.

\section{References}

[1] W. Zhang, L. Q. Huang, C. X. Li et al., "Literature study on the species of honeysuckle flower," Journal of Chinese Materia Medica, vol. 39, no. 12, pp. 2239-2244, 2014.

[2] S.-H. Zhang, Q.-Z. Yuan, G.-X. Cai, X.-Y. Feng, and Y. Shao, "Fingerprint of different polarity extracts from superfine powder of Lonicera japonica and its correlation of antibacterial activity," Chinese Traditional and Herbal Drugs, vol. 42, no. 11, pp. 2226-2230, 2011.

[3] J. Sun, Ethanol extracts of honeysuckle and the in vitro inhibitory effects on Enterococcus faecalis biofilm in root canal [M.S. thesis], Jiamusi University, Jiamusi, China, 2010.

[4] Y. Xu, D. C. Li, and J. Sun, "Antibacterial effect of honeysuckle ethanol extracts on enterococcus faecalis biofilm in root canal: 
an in vitro study," Heilongjiang Medicine and Pharmacy, vol. 33, no. 6, pp. 49-50, 2010.

[5] S. X. Wang and X. Yang, "Ethanol extracts of Lonicera japonica and the in vitro inhibitory effects on the Enterococcus faecalis biofilm in root canal," Journal of Qiqihar University of Medicine, vol. 35, no. 9, pp. 1277-1279, 2014.

[6] P. Li and C. Zhao, "Antibacteria test of aqueous extract and ethanol extract of Lonicerae japonicae flos," China Mordern Medicine, vol. 17, no. 17, pp. 11-14, 2010.

[7] C. L. He, X. Y. Zhang, H. Yang et al., "Antibacterial activity of Loniceraeflos extract to Staphylococcus aureus," Guizhou Journal of Animal Husbandry \& Veterinary Medicine, vol. 37, no. 4, pp. 11-14, 2013.

[8] Q. Wang, X. X. Zhu, C. B. Zhang et al., "Experimental study on honeysuckle extract against bacteria," Chinese Journal of Medical Guide, vol. 10, no. 9, pp. 1428-1430, 2008.

[9] Z. Y. Guan, J. M. Zhao, and Q. Z. Lin, "The effect of extract of honeysuckle on bactriostasis," China Modern Doctor, vol. 47, no. 15, pp. 150-151, 153, 2009.

[10] J. Liu, "The in vivo study of anti-bacterial and anti-inflammatory activities of honeysuckle extracts in mouse," Modern Medicine Journal of China, vol. 11, no. 3, pp. 127-128, 2009.

[11] X. Ren, Q. Li, W. B. Li et al., "The study of anti-bacterial activities of 15 medicinal plants of Lonicera L.", Territory \& Natural Resources Study, vol. 2, pp. 68-70, 1991.

[12] D. M. Liu, J. C. Bi, and H. Q. Qie, "Antimicrobial activity of Scutellaria baicalensis Georgi, Coptis chinensis Franch, Prunus mume Sieb, Lonicera japonica Thunb, Patrinia scabiosaefolia Fisch on bacteria producing AmpC $\beta$-lactamases," Hebei Journal of Traditional Chinese Medicine, vol. 30, no. 6, pp. 654-656, 2008.

[13] X. P. Su, B. W. Song, S. H. Chen et al., "Study on the volatile oil of Lonicerae flos by supercritical $\mathrm{CO}_{2}$ extraction and its antiinflammatory activity," Natural Product Research and Development, vol. 18, no. 4, pp. 663-666, 2006.

[14] X. P. Su, D. C. Gong, Y. W. Zhang et al., "Research on the growth inhibition of $\mathrm{CO}_{2}$-SFE extracts from Lonicerae on bacteria in vitro," Lishizhen Medicine and Materia Medica Research, vol. 20, no. 4, pp. 832-834, 2009.

[15] L. Z. Zhao, L. D. Duan, and Y. G. Yu, "Study on honeysuckle antimicrobial extraction and antimicrobial effect," Journal of Shaoyang College, vol. 14, no. 3, pp. 204-209, 2001.

[16] X. P. Lin, X. Q. Chen, M. C. Su et al., "Antimicrobial activities of polysaccharide extracts from Lonicerae flos and Ilex kudingcha," Subtropical Plant Science, vol. 37, no. 1, pp. 51-53, 2008.

[17] J. Yu, X. J. Su, L. Wang et al., "Antifungal effects of eight kinds of traditional Chinese medicine in vitro," Academic Journal of PLA Postgraduate Medical School, vol. 28, no. 4, pp. 299-301, 2007.

[18] L. Q. Wang, B. A. Cui, and H. Y. Zhang, "Pharmacological action and research progress of Lonicerae flos," China Animal Husbandry \& Veterinary Medicine, vol. 34, no. 11, pp. 91-95, 2007.

[19] Y. Wang, J. Yu, Y. Xiao et al., "Studies on the elimination of resistance plasmid from $P$. aeruginosa in vitro and vivo with Lonicera japonica," Journal of Norman Bethune University of Medical Science, vol. 26, no. 2, pp. 139-140, 2000.

[20] Y. Feng and H. L. Wang, "A study on Lonicerae flos' medicinal component and pharmacological effect," Clinical Journal of Chinese Medicine, vol. 6, pp. 95-96, 2014.

[21] B. W. Xia, "Review about honeysuckle," Chinese Journal of Ethnomedicine and Ethnopharmacy, vol. 2, pp. 88-89, 2003.
[22] K. S. Chen, Q. Cai, X. L. Lv et al., "Experimental study of the effects of Coptis roots, Lonicerae japonicae flos and Houttuynia cordata on the positive coccus," Practical Clinical Journal of Integrated Traditional Chinese and Western Medicine, vol. 9, no. 3, pp. 87-88, 2009.

[23] J. L. Liu, The study on the relationship of geo-herbalism and efficacy of Lonicerae flos in Guangxi [M.D thesis], University of Guangxi, 2009.

[24] Y. Zhu and Z. L. Xu, "Studies on correlation between bacteriostasis and total flavonoids, chlorogenic acid content in leaves of Lonicera hypoglauca Miq," Chinese Journal of Traditional Medical Science and Technology, vol. 21, no. 1, pp. 39-41, 2014.

[25] J. L. Li, Q. Tang, G. Chen et al., "Study on the bacteriostatic activity, anti-inflammation, analgesic and antipyretic effects of extract from Lonicera bud," Science and Technology of Food Industry, vol. 33, no. 19, pp. 82-87, 2012.

[26] L. N. Chen, "Study on the antibacterial effect of Lonicerae flos," Clinical Medical Engineering, vol. 16, no. 10, pp. 46-47, 2009.

[27] Z. J. Lei, Comparative Study and Safty Evaluation of the L.macranthodes Hand-Mazz and Lonicera japonica Thunb, TCM University of Hunan, 2003.

[28] Z. P. Gou and D. G. Wan, "Antibacterial activity of varieties of Lonicerae flos produced in Sichuan in vitro by beating holes method," Lishizhen Medicine and Materia and Medica Research, vol. 19, no. 3, pp. 724-725, 2008.

[29] L. Liu and R. Li, "Extract of ingredients for medicine in Lonicera confusa DC and study on antibiotic activity," Journal of Medical Science in Central South China, vol. 40, no. 3, pp. 298-300, 2012.

[30] Y. Liu and G. Wang, "Effect of Lonicerae flos extracts on herpes simplex keratitis," Herald of Medicine, vol. 30, no. 11, pp. 14211424, 2011.

[31] Z. Pan, X. Wang, L. Yan et al., "Inhibitory effect of extracts from Lonicerae flos on influenza a virus FM1 strain in vitro," Chinese Journal of Information on Traditional Chinese Medicine, vol. 14, no. 6, pp. 37-38, 51, 2007.

[32] Z. Ji, X. Zhu, W. Ni et al., "Studies on the antiviral effect of Lonicerae flos extracts," Chinese Journal of Medical Guide, vol. 11, no. 1, pp. 92-93, 2009.

[33] J. Shi and C. Guo, "Study on effective fraction of anti-influenza virus of Lonicerae japonicae flos in vitro," Journal of Shandong University of Traditional Chinese Medicine, vol. 34, no. 2, pp. 178-180, 2010.

[34] F. Cheng, Y. Jiang, Y. Yu et al., "Study on anti-prrsv effect of Loniera japonica extracts in vitro," Progress in Veterinary Medicine, vol. 33, no. 2, pp. 41-45, 2012.

[35] R. Zhang, Y. Hua, and D. Liu, "Study on the antiviral effects of Lonicerae flos against Newcastle disease in chick embryo," Journal of Traditional Chinese Veterinary Medicine, vol. 4, no. 4, pp. 33-36, 2010.

[36] K. Hu, Y. Wang, and D. Wang, "The inhibited effect of chlorogenic acid from the honeysuckle on virus in vitro," Information on Traditional Chinese Medicine, vol. 27, no. 3, pp. 27-28, 2010.

[37] M. Li, "In vitro anti-respiratory syncytial virus effect of the extraction of Lonicera japonica Thunb," Journal of Tropical Medicine, vol. 10, no. 4, pp. 420-422, 2010.

[38] D.-Q. Yu, R.-Y. Chen, L.-J. Huang et al., "The structure and absolute configuration of Shuangkangsu: a novel natural cyclic peroxide from Lonicera japonica (Thunb.)," Journal of Asian Natural Products Research, vol. 10, no. 9, pp. 851-856, 2008.

[39] Y. Kashiwada, Y. Omichi, S.-I. Kurimoto et al., "Conjugates of a secoiridoid glucoside with a phenolic glucoside from the flower 
buds of Lonicera japonica Thunb," Phytochemistry, vol. 96, pp. 423-429, 2013.

[40] J.-J. Chen, J.-Q. Fang, J. Wan et al., "An in vitro study of the anticytomegalovirus effect of chlorogenic acid," Herald of Medicine, vol. 28, no. 9, pp. 1138-1141, 2009.

[41] X. Wang, S. Chen, F. Qiao et al., "Experimental study of Honeysuckle flower against guinea pig Cytomegalovirus in vitro," Maternal and Child Health Care of China, vol. 20, no. 20, pp. 2241-2243, 2005.

[42] L. Wang, B. Cui, and H. Zhang, "Antiviral effect of Lonicerae japonica flos and Lonicerae flos flavonoids ingredients to PRV in vitro," China Animal Husbandry \& Veterinary Medicine, vol. 38, no. 3, pp. 183-188, 2011.

[43] J. Dong, C. Chen, S. Qiu et al., "Experimental study on anti-coxsackie and ECHO virus of 4 TCM herbs," Journal of Shandong University of Traditional Chinese Medicine, vol. 17, no. 4, pp. 46-48, 2011.

[44] X. Shen, X. Luo, and N. Li, "Molecular mechanism study on anti-influenza of Lonicerae japonica flos," Shaanxi Journal of Traditional Chinese Medicine, vol. 33, no. 9, pp. 1247-1248, 2012.

[45] Y. M. Li, L. Li, C. Bai et al., "Effect of extracts from honeysuckle flower on anti-adenovirus," West China Journal of Pharmaceutical Sciences, vol. 16, no. 5, pp. 327-329, 2001.

[46] X. Bao, C. Guo, and F. Wang, "Preliminary research of Lonicerae japonica flos on protective effect against mouse's lung injury induced by influenza virus," Lishizhen Medicine and Materia Medica Research, vol. 24, no. 3, pp. 583-584, 2013.

[47] S.-Y. Wang, C.-P. Tseng, K.-C. Tsai et al., "Bioactivity-guided screening identifies pheophytina as a potent anti-hepatitis $\mathrm{C}$ virus compound from Lonicera hypoglauca Miq.", Biochemical and Biophysical Research Communications, vol. 385, no. 2, pp. 230-235, 2009.

[48] L. Wang, H. Zhang, B. Cui et al., "The study on antiviral effect of chlorogenic acids from Lonicerae japonica flos and Lonicerae flos on NDV in vitro," Chinese Agricultural Science Bulletin, vol. 27, no. 19, pp. 277-282, 2011.

[49] L. Q. Wang, Studies on antiviral and immunne-enhancing activity of Lonicerae japonica flos and Lonicerae flos [M.S. thesis], Henan Agricultural University, Zhengzhou, China, 2008.

[50] X. Y. Cui, S. X. Wang, and Y. L. Hou, "Anti-inflammatory mechanism of the Lonicera japonica Thunb extract," China Pharmacy, vol. 18, no. 24, pp. 1861-1863, 2007.

[51] X. G. Zhou, S. L. Liang, C. H. Hu et al., "Inhibitory effect on acute inflammation of ethanol extract from Lonicera japonica," Pratical Clinical Medicine, vol. 13, no. 2, pp. 12-21, 2012.

[52] P. F. Li, H. Guan, and C. Y. He, "The anti-inflammatory action of four kinds of medicinal herbs," Journal of Inner Mongolia College of Agriculture \& Animal Husbandry, vol. 11, no. 1, pp. 36-39, 1990.

[53] L. Q. Wang, B. A. Cui, H. Y. Zhang, W.-M. Gao, and S.-L. $\mathrm{Li}$, "Study on the anti-inflammatory activity of the product extrouted from Lonicerae japonica flos," China Animal Husbandry \& Veterinary Medicine, vol. 35, no. 8, pp. 82-84, 2008.

[54] P. Wang and X. L. Zhang, "The treatment of cervicitis in rats from Lonicera japonica," China Journal of Gerontology, vol. 32, no. 7, pp. 1441-1443, 2012.

[55] C. F. Zheng, Study on compounds and antivirus bioactivities from Lonicera japonica and morus Australia [Ph.D. thesis], Chinese Academy of Medical Sciences \& Peking Union Medical College, 2010.
[56] S. W. Li, J. J. Gu, and L. Zhao, "Protective mechanism of Lonicerae flos and fructus forsy thiae for mouse infected by Escherichia coli," Modern Journal of Integrated Traditional Chinese and Western Medicine, vol. 22, no. 6, pp. 594-596, 2013.

[57] W.-C. Chen, S.-S. Liou, T.-F. Tzeng, S.-L. Lee, and I.-M. Liu, "Wound repair and anti-inflammatory potential of Lonicera japonica in excision wound-induced rats," BMC Complementary and Alternative Medicine, vol. 12, article 226, 2012.

[58] S. H. Hong, J. T. Kwon, J. Y. Shin et al., "Therapeutic effect of Broussonetia papyrifera and Lonicera japonica in ovalbumininduced murine asthma model," Natural Product Communications, vol. 8, no. 11, pp. 1609-1614, 2013.

[59] Y. Inami, Y. Matsui, T. Hoshino, C. Murayama, and H. Norimoto, "Inhibitory activity of the flower buds of Lonicera japonica thunb. Against histamine production and L-histidine decarboxylase in human keratinocytes," Molecules, vol. 19, no. 6, pp. 8212-8219, 2014.

[60] E. Park, S. Kum, C. Wang, S. Y. Park, B. S. Kim, and G. SchullerLevis, "Anti-inflammatory activity of herbal medicines: inhibition of nitric oxide production and tumor necrosis factor$\alpha$ secretion in an activated macrophage-like cell line," The American Journal of Chinese Medicine, vol. 33, no. 3, pp. 415424, 2005.

[61] O.-H. Kang, Y.-A. Choi, H.-J. Park et al., "Inhibition of trypsininduced mast cell activation by water fraction of Lonicera japonica," Archives of Pharmacal Research, vol. 27, no. 11, pp. 1141-1146, 2004.

[62] K.-I. Park, S.-R. Kang, H.-S. Park et al., "Regulation of proinflammatory mediators via NF- $\kappa \mathrm{B}$ and p38 MAPK-dependent mechanisms in RAW 264.7 macrophages by polyphenol components isolated from Korea Lonicera japonicaTHUNB," Evidence-Based Complementary and Alternative Medicine, vol. 2012, Article ID 828521, 10 pages, 2012.

[63] M. Jiang, Y.-Q. Han, M.-G. Zhou et al., “The screening research of anti-inflammatory bioactive markers from different flowering phases of Flos Lonicerae Japonicae," PLoS ONE, vol. 9, no. 5, Article ID e96214, 2014.

[64] Y. Zhu and S. S. Li, "Experimental study of anti-inflammatory and antipyretic effect on leaves of Lonicera hypoglauca Miq," Journal of Gansu College of TCM, vol. 31, no. 3, pp. 12-17, 2014.

[65] Y. F. Hu and S. H. Li, "Anti-inflammatory mechanism of the volatile oil extract from the Lonicera macran thoides," Lishizhen Medicine and Materia Medica Research, vol. 23, no. 12, pp. 30313035, 2012.

[66] Y. F. Hu, The research on the flower of Lonicera macran thoidesvolatileorganic compounds and the ecological health effects [Ph.D. thesis], Central South University of Forestry \& Technology, 2012.

[67] L. Wang, Study of effective substances screening for Lonicerae flos based on the 'spectrum-effect' combination [M.S. thesis], Chengdu University of Traditional Chinese Medicine, Chengdu, China, 2010.

[68] Y. D. Luo and Y. M. Luo, "Study on the pharmacodynamics of the antioxidant activity of the flavonoid effective fractions from Lonicera japonica," Journal of Jiangxi University of Traditional Chinese Medicine, vol. 23, no. 2, pp. 47-49, 2011.

[69] Y. F. Ma, "Study on antioxidative effect of Lonicera simlis Hemsl," Journal of Anhui Agricultural Sciences, vol. 35, no. 11, pp. 32413242, 2007.

[70] C. P. Liu, "Analysis on anti-oxidized activity of flavonoid from Honeysuckle," Journal of Anhui Agricultural Sciences, vol. 37, no. 20, pp. 9483-9484, 9505, 2009. 
[71] J. H. Song, "Study on antipyretic and anti-inflammatory effect of Lonicera japonica," Chongqing Medical Journal, vol. 40, no. 25, pp. 2552-2553, 2011.

[72] X. Y. Cu and M. Dong, "Effect of Lonicera japonica Thumbcontaining-serum on releasing of no from normal and lipopolysaccharide induced primary rats microglia cells," Journal of Hebei Medical University, vol. 29, no. 2, pp. 245-248, 2008.

[73] Z. B. Zhu, B. Tian, and J. H. Yi, "Comparative study on the antioxidation activity of Lonicerae flos extracts using water and ethanol," Food and Fermentation Industries, vol. 34, no. 2, pp. 69-72, 2008.

[74] Y. Teng, X. Wang, X. L. Feng et al., "Scavenging effects of different ethanol extracts from Lonicera japonica on DPPH free radical S by EPR," Heilongjiang Medicine and Pharmacy, vol. 34, no. 6, pp. 44-46, 2011.

[75] X. L. Chen and Y. R. Ren, "Study on anti-oxidation of waterextract from honeysuckle," Lishizhen Medicine and Materia Medica Research, vol. 21, no. 7, pp. 1652-1653, 2010.

[76] R. W. Li, Study on chromatographic analysis of honeysuekle and propolis and evaluation of their antioxidant activities [M.S. thesis], Northwest University, Kirkland, Wash, USA, 2008.

[77] M. Wang, F. Liu, C. H. Lin et al., "Impacts of different drying methods on antioxidant activity and chemical ingredients of honeysuckle by chemiluminescence detection," Shandong Science, vol. 26, no. 2, pp. 56-65, 2013.

[78] Y.-S. Chen, H.-C. Liou, and C.-F. Chan, "Tyrosinase inhibitory effect and antioxidative activities of fermented and ethanol extracts of Rhodiola rosea and Lonicera japonica," The Scientific World Journal, vol. 2013, Article ID 612739, 5 pages, 2013.

[79] C.-C. Gong, N.-G. Zheng, J.-L. Wu, Z.-R. Lai, and P.-X. Hen, "Antioxidant effect of honeysuckle on hepatic RBL cells of rats in vivo and in vitro and mechanism," Journal of Jilin University Medicine Edition, vol. 35, no. 6, pp. 1074-1078, 2009.

[80] M. L. Men, C. C. Gong, Y. X. Zheng et al., "The molecular mechanism of antioxidative effects of Honeysuckle," Practical Journal of Medicine \& Pharmacy, vol. 25, no. 9, pp. 1104-1106, 2008.

[81] Y. Y. Zhang, Y. H. Wang, Y. Shi, Y. H. Wang, and Y. Y. Zhang, "Antioxidant effect of Lonicera japonica extract in D-galactose induced aging model mice," Journal of Zhejiang Chinese Medical University, vol. 38, no. 3, pp. 321-326, 2014.

[82] X. Zhu, "Pharmacological effects and clinical situation analysis of Lonicerae japonicae flos," Medicine and Health Care, vol. 22, no. 5, pp. 64-65, 2014.

[83] X. Xie, S. Jiang, X. Zou, Q. Zhou, E.-S. Wen, and J.-H. Xue, "Study on antipyretic effect and mechanism of Jinyinhua in pyretic rabbits," Lishizhen Medicine and Materia Medica Research, vol. 20, no. 3, pp. 691-692, 2009.

[84] H. Duan and M. Cheng, "Experimental study on pyretolysis mechanism of compatible application of Japanese honeysuckle flower bud and weeping Forsythia fruit," Modern Journal of Integrated Traditional Chinese and Western Medicine, vol. 18, no. 11, pp. 1214-1216, 2009.

[85] J. L. Li, Q. Tang, G. Chen et al., "Study on the bacteriostatic activity, anti-inflammation, analgesic and antipyretic effects of extract from Lonicera bud," Science and Technology of Food Industry, vol. 33, no. 19, pp. 82-87, 2012.

[86] Z. Lei, R. Zhou, and Q. Tong, "Security compare of Lonicera macranthoides Hand-Mazz and Lonicerae japonicae flos," Chinese Traditional Patent Medicine, vol. 28, no. 5, pp. 759-761, 2006.
[87] C. Zhou, Y. Su, and F. Li, "Protective effect of honeysuckle extracts on chemically-induced injury in mice liver," Modern Food Science and Technology, vol. 26, no. 4, pp. 351-353, 2010.

[88] H. Chen, "Effect of Jinyinghua on carbon tetrachloride induced acute hepatic injury," Chinese Journal of Gerontology, vol. 31, no. 8, pp. 3086-3087, 2011.

[89] J. Shi, L. Wan, and X. Chen, "Protective effect of total saponins of Lonicera macranthoides Hand-Mazz on several toxic chemicals liver injury mice," Pharmacology and Clinics of Chinese Materia Medica, vol. 6, no. 1, pp. 33-34, 1990.

[90] J. Shi, X. Chen, and L. Wan, "Hepato protective effect of several constituents of Lonicera fulvotomentosa Hsu et S. C. Cheng, and L .macranthoide Hand-Mazz on CC14 and D-galactosamine induced liver injuries in mice and rats," China Journal of Chinese Materia Medica, vol. 24, no. 6, pp. 43-64, 1999.

[91] Q. Xue, Y. Liu, Y.-J. Li, L. Li, S.-H. Wang, and X.-Y. Xu, “The immunomodulatory and the protection of experimental liver injury of Lonicera macranthoides Hand-Mazz on mice," Chinese Pharmaceutical Journal, vol. 48, no. 8, pp. 601-606, 2013.

[92] Y.-P. Liu, J. Liu, X.-S. Jia, Q. Mao, C. Madhu, and C. D. Klaassen, "Protective effects of fulvotomentosides on acetaminopheninduced hepatotoxicity," Acta Pharmacologica Sinica, vol. 13, no. 3, pp. 209-212, 1992.

[93] M. Chen and Q. Liu, "Medicinal composition and pharmacological effect of Lonicerae flos," Journal of North Pharmacy, vol. 10, no. 10, p. 29, 2013.

[94] S. Wu and D. Zhai, "Review of chemical component and pharmacologcacton of Lonicerae flos," Science and Technology Innovation Herald, vol. 20, no. 20, p. 2, 2008.

[95] X. Zhou, Z. Li, Z. Liu et al., "Effect of Lonicerae flos extract made by different methods on immunologic function of rats," Practical Preventive Medicine, vol. 18, no. 11, pp. 2052-2054, 2011.

[96] S. Chang, "Review of pharmacology research of Lonicerae flos," Chinese Journal of Ethnomedicine and Ethnopharmacy, vol. 1, no. 1, p. 49, 2010.

[97] C. Hu, J. Hu, H. Liu et al., "Protective effect of Lonicera japonica total flavone (LJTF ) on immunological liver injury in $\mathrm{m}$ ice," Pharmacology and Clinics of Chinese Materia Medica, vol. 23, no. 5, pp. 85-88, 2007.

[98] B. Liu and Y. Liu, "Proliferation effects of Lonicerae flos polysaccharide on spleen lymphocytes," China Practical Medicine, vol. 8, no. 11, pp. 244-245, 2013.

[99] H. Yin, X. Lv, and X. Wei, "Study on process optimization and immune activity of Lonicerae flos polysaccharide," China Journal of Chinese Materia Medica, vol. 35, no. 4, pp. 453-455, 2010.

[100] Z. H. Luo, "The combined modulating effects of cerium nitrate with certain Chinese traditional drugs on altered cell-mediated immunities in scald mice," Chinese Journal of Surgery, vol. 28, no. 9, pp. 562-574, 1990.

[101] H. Hou, Y. Zeng, X. Huang, and L.-L. Yin, "Effects of Lonicerae flos extracts on the activation and proliferation of mouse lymphocytes in vitro," Immunological Journal, vol. 24, no. 2, pp. 178-183, 2009.

[102] X. N. Zhang, Q. Tang, M. Hou et al., "Comparison of the pharmacological effects between L. macranthoides Hand.-Mazz and Lonicera japonica Thunb," Chinese Pharmacological Bulletin, vol. 28, no. 11, pp. 1582-1585, 2012.

[103] Y. G. Liu, Y. H. Liu, and H. Q. Jiang, "Inhibitory effect and mechanism of polysaccharide from Lonicera japonica on mice bearing S180 sarcoma," Journal of Chinese Oncology, vol. 18, no. 8, pp. 584-587, 2012. 
[104] H.-S. Park, K.-I. Park, D.-H. Lee et al., "Polyphenolic extract isolated from Korean Lonicera japonica Thunb. induce G2/M cell cycle arrest and apoptosis in HepG2 cells: involvements of PI3K/Akt and MAPKs," Food and Chemical Toxicology, vol. 50, no. 7, pp. 2407-2416, 2012.

[105] Y. Chen, X. Z. Zhao, and X. D. Jia, "Chemical ingredients of macranthoides and progress in anti-cancer," in Proceedings of the 8th National Symposium on Natural Medicinal Material Resources Proceedings and Abstracts, pp. 6-7, 2008.

[106] F. Q. Guan, X. Feng, F. Peng et al., "Antiproliferative effect of macranthoside B on leukemia cell lines HL-60 and its mechanism," Natural Product Research and Development, no. 22, pp. 765-768, 811, 2010.

[107] F. Guan, Y. Shan, X. Zhao et al., "Apoptosis and membrane permeabilisation induced by macranthoside B on HL-60 cells," Natural Product Research, vol. 25, no. 4, pp. 332-340, 2011.

[108] W. G. Ren, S. S. Lin, W. T. Li, and L. F. Huang, "Analysis of chemical constituents of the effective part of anti-EGFR of Lonicera macranthoides Hand-Mazz by UPLC-Q-TOF-MS," Journal of China Pharmaceutical University, vol. 44, no. 6, pp. 526-530, 2013.

[109] J. Q. Pan, H. C. Liu, and G. N. Liu, "Honeysuckle can reduce the level of blood glucose and blood lipid of mice," Guangzhou Pharmaceuticals, vol. 29, no. 3, pp. 59-61, 1998.

[110] X. L. Chen, "Effects of water extract from honeysuckle on glycometabolism in vitro," Lishizhen Medicine and Materia Medica Research, vol. 21, no. 3, pp. 628-629, 2010.

[111] Q. Wang, D. H. Chen, and W. L. Deng, "Effect of extracts from Lonicerae flos on blood lipid and blood glucose," Pharmacology and Clinics of Chinese Materia Medica, vol. 23, no. 3, pp. 40-42, 2007.

[112] R. Li, Y. Zhou, S. Y. Kuang, C. Wang, and J. Wang, "Study on the anti-atherosclerosis effect of Lonicera macranthoides Hand-Mazz. extract," The Chinese Journal of Modern Applied Pharmacy, vol. 28, no. 2, pp. 92-95, 2011.

[113] H. Oku, Y. Ogawa, E. Iwaoka, and K. Ishiguro, "Allergy-preventive effects of chlorogenic acid and iridoid derivatives from flower buds of Lonicera japonica," Biological and Pharmaceutical Bulletin, vol. 34, no. 8, pp. 1330-1333, 2011.

[114] F. Li, "The study of Lonicera extract from water solution on ovalbumin-induced allergic mice model," Journal of Chongqing Medical University, vol. 29, no. 3, pp. 288-291, 2004.

[115] F. Li and H. Li, "Effects of Lonicera on the immunoregulation of intestinal mucosal inflammatory response and sIgA response in ovalbumin sensitized BALB/c mice," Journal of Clinical Pediatrics, vol. 25, no. 7, pp. 585-589, 2007.

[116] F. Bai and H. Q. Li, "Antiallergic effects of fulvotomento-side on ovalbum in-sensitized BALB/c mice," Journal of the Fourth Military Medical University, vol. 29, no. 15, pp. 1395-1398, 2008.

[117] F. Bai, Immunoregulatory effects of fulvotomentoside on ovalbumin-sensitized BALB/c mice [Ph.D. thesis], Chongqing Medical University, Chongqing, China, 2009.

[118] Y. F. Hu and S. H. Li, "Gray wool felt on anti-inflammatory effects and mechanism of honeysuckles of volatile organic compounds," Lishizhen Medicine and Materia Medica Research, vol. 23, no. 12, pp. 3031-3035, 2012.

[119] C. P. Cao, Z. N. Huang, and P. L. Qian, "Study on antifertility effect of honeysuckle," Pharmaceutical Industry, vol. 17, no. 3, pp. 115-117, 1986.

[120] C. J. Yang, D. W. Su, Y. S. Wang, K. Zhang, S. X. Ma, and L. Y. Yang, "The effect of Chinese medicine double flower on intestinal microflora dysbiosis," Chinese Journal of Microecology, vol. 24, no. 2, pp. 132-138, 2012.

[121] Y. C. Ran, H. Q. Li, and M. F. Liu, "Honeysuckle extract on traditional Chinese medicine pharmacology and clinical effects of growth of Bifidobacterium and Lactobacillus," Pharmacology and Clinics of Chinese Materia Medica, no. 5, pp. 118-120, 2007.

[122] C. E. Feng, R. X. Li, and Q. F. Huang, "Protective effect on the skin of mice after exposure to UVA," Asia-Pacific Traditional Medicine, vol. 10, no. 12, pp. 3-5, 2014.

[123] L. Lei, X. P. Li, X. L. Bai et al., "The anti-endotoxin, antipyretic, anti-inflammatory effects of Lonicera japonica," Pharmacology and Clinics of Chinese Materia Medica, vol. 28, no. 1, pp. 115-117, 2012.

[124] S.-J. Song, F.-F. Li, Y. Xu, L.-H. Zhang, F. Yuan, and Y. Zhang, "Effects of Honeysuckle flower and Scutellaria Baicalensis Georgi on constraction and electric activity of rabbit small intestine smooth muscle," Chinese Journal of Applied Physiology, vol. 25, no. 3, pp. 344-348, 2009.

[125] M. Wang, S. Ni, S. Liu et al., "Effect of Honeysuckle preparation decocted with water and deposited with alcohol on in vitro small intestine movement of domestic rabbit," Chinese Agricultural Science Bulletin, vol. 21, no. 6, pp. 32-34, 2005.

[126] H. Fan, D. Xiao, L. Yu, and Q. Zhu, "In vitro antiplatelet aggregative effects of honeysuckle and its organic acid compounds," Chinese Journal of Hospital Pharmacy, vol. 26, no. 2, pp. 145-147, 2006.

[127] L. Ji, J. Pan, and Z. Xu, "GC-MS analysis of essential oil from flowers of Lonicera japonica Thunb," China Journal of Chinese Materia Medica, vol. 15, no. 11, pp. 680-703, 1990.

[128] W.-C. Chang and F.-L. Hsu, "Inhibition of platelet activation and endothelial cell injury by polyphenolic compounds isolated from Lonicera japonica Thunb," Prostaglandins, Leukotrienes and Essential Fatty Acids, vol. 45, no. 4, pp. 307-312, 1992.

[129] S.-H. Kwon, S.-I. Hong, Y.-H. Jung et al., "Lonicera japonica THUNB. protects 6-hydroxydopamine-induced neurotoxicity by inhibiting activation of MAPKs, PI3K/Akt, and NF- $\kappa \mathrm{B}$ in SH-SY5Y cells," Food and Chemical Toxicology, vol. 50, no. 3-4, pp. 797-807, 2012.

[130] S.-H. Kwon, S.-I. Hong, J.-A. Kim et al., "The neuroprotective effects of Lonicera japonica THUNB. against hydrogen peroxide-induced apoptosis via phosphorylation of MAPKs and PI3K/Akt in SH-SY5Y cells," Food and Chemical Toxicology, vol. 49, no. 4, pp. 1011-1019, 2011.

[131] P. Wang, W. Liao, J. Fang et al., "A glucan isolated from flowers of Lonicera japonica Thunb. Inhibits aggregation and neurotoxicity of A $\beta_{42}$," Carbohydrate Polymers, vol. 110, pp. 142147, 2014.

[132] Y. Shi, R. B. Shi, and Y. R. Lu, "Studies on medicinal resource, phytochemistry and pharmacology of Lonicerae flos," Chinese Pharmaceutical Journal, vol. 34, no. 11, pp. 724-727, 1991.

[133] J. Zhang, P. P. Shen, and X. M. Zhang, "Toxicological assesment of the edible safety of Lonicerae flos," The Chinese Academic Medical Magazine of Organisms, vol. 22, no. 2, pp. 63-64, 2003.

[134] H. Li, L. H. Zhang, and H. P. Wang, "Primary study on antipyretic, anti-inflammatory actions and acute toxicity of Lonicera similis Hemsl," Strait Pharmaceutical Journal, vol. 20, no. 9, pp. 28-31, 2008.

[135] J. L. Li, H. Zhang, G. Chen et al., "Toxicological evaluation on Lonicera bud," Science and Technology of Food Industry, vol. 33, no. 23, pp. 355-357, 361, 2012. 
[136] F. J. Jiang, Studies on antibacterial action in vitro and antioxidation of extract of Lonicerae flos Xianglei the antibacterial effect [M.S. thesis], Hunan Agricultural University, Changsha, China, 2006.

[137] Q. P. Pan, Z. J. Lei, R. B. Zhou et al., "Comparative experiments on bacteriostasis between Lonicera macrathodes Hands Mazz. and the certified Lonicera flos," Chinese Archives of Traditional Chinese Medicine, vol. 22, no. 2, pp. 243-244, 2004.

[138] M. Hou, Q. Tang, X.-N. Zhang, Y. Li, and X.-Y. Xu, “Comparative studies on pharmacodynamics of Lonicera macranthoides and Lonicera japonica," Chinese Traditional and Herbal Drugs, vol. 44, no. 3, pp. 309-314, 2013.

[139] Q. Li and Y. Cui, "A comparison on pharmacological actions of Lonicera japonica," Journal of Chinese Medicinal Materials, vol. 22, no. 1, pp. 37-39, 1999.

[140] Z. J. Lei, R. B. Zhou, R. Zeng et al., "Compare on antibacterial action between large flower-like honeysuckle flower and the certified honeysuckble," Journal of Traditional Chinese Medicine University of Hunan, vol. 11, no. 9, pp. 8-10, 2005.

[141] H. J. Li, P. Li, Z. Y. Zhang et al., "Lonicera japonica thunb extract on the effect of free radical scavenging," Journal of China Pharmaceutical University, vol. 22, no. 6, pp. 42-44, 2002.

[142] L. P. Wang, N. Xin, Y. Qiu et al., "Antioxidative effects of total flavonoids from Guangxilonicera hypo glaucamip and Shandong lonicera japonica in vivo," Journal of Guangxi Medical University, vol. 27, no. 5, pp. 681-683, 2010.

[143] F. Li and H.-Q. Li, "Immunoregulatory effects of the Lonicera aquatic extract in the ovalbulmin-sensitized BALB/c mice," Chinese Journal of Pediatrics, vol. 43, no. 11, pp. 57-62, 2005.

[144] Y. Y. Wang, S. Q. Chang, Y. Q. Li et al., "Study on the quality of Lonicera macranthoides (III)-effects of different processing methods tonoxicities and haemolyticus," Chinese Herbal Medicines, vol. 7, no. 2, pp. 17-18, 1984.

[145] W. Y. Feng, M. H. Liu, S. H. Xiao et al., "Experimental study on the active systematic anaphylax is of honeysuckle refined extract and crude extract injection," Lishizhen Medicine and Materia Medica Research, vol. 19, no. 12, pp. 2847-2848, 2008.

[146] J. H. Liu, The Study on Lonicerae japonicae Flos and Lonicerae flos on quality evaluation and comparative study on safety [Ph.D. thesis], Beijing University of Chinese Medicine, Beijing, China, 2014.

[147] Y. B. Sun, Y. Wang, X. Z. Guan, and Y. B. Sun, "A study on antimicrobial properties of Lonicerae flos against oral pathogens," China Journal of Chinese Materia Medica, vol. 37, no. 8, pp. 242-244, 1996.

[148] L. Z. Zhao, X. Y. Jiang, L. D. Yin et al., “The extraction and antibacterial activity identification of the water-soluble substances in Lonicera japonica Thunb," Chinese Journal of Biologicals, vol. 19, no. 2, pp. 201-203, 2006.

[149] L. P. Yu and L. W. Cai, "Detect of antisepsis ability of Honeysuckle flower, Ilex latifolia thunb, wild Chrysanthemum flower and Cassia seed," Journal of Hangzhou Medical college, vol. 24, no. 2, pp. 77-78, 2013.

[150] X. L. Yuan, J. L. Lv, and H. J. Chen, "The synergetic effects of Gentamicin and water extracts from honey suckle flower in the inhibition of Pseudomonas aeruginosa biofilm formation," Northwest Pharmaceutical Journal, vol. 25, no. 3, pp. 201-203, 2010.

[151] Y. Z. Cui, X. Wang, T. S. Han et al., "Experimental study and the dose-efficacy relationship analysis of the enhancement effects of TMP for the anti-bacterial activity of honey suckle flower,"
Chinese Journal of Veterinary Medicine, vol. 46, no. 8, pp. 60-61, 2010.

[152] C. H. Wang and H. J. Wang, "The antibacterial action of five traditional Chinese herbs including radix glycyrrhiza and rhizomacoptidis," Chinese Journal of Veterinary Medicine, vol. 50, no. 3, pp. 44-46, 2014.

[153] T. T. Zhang, Y. Y. Zhang, H. G. Chen et al., "Establishment of a novel preliminary screening model for bioactive parts of Lonicerae japonicae Thunb based on microcalorimetry," Pharmaceutical Journal of Chinese People's Liberation Army, vol. 27, no. 3, pp. 205-211, 2011.

[154] L. Li and J. Sun, "Comparative on content of chlorogenic acid and bacteriostasis of extracts from different parts of polyploid and diploid Honeysuckle," Food Science and Technology, vol. 37, no. 8, pp. 225-231, 2012.

[155] L. Li, Y. J. Li, S. H. Wang et al., "Antibacterial and anti-inflammatory effect of L. macranthoides Hand-Mazz'salabastrum," Science and Technology of Food Industry, vol. 34, no. 23, pp. 6569, 2013.

[156] H.-C. Ko, B.-L. Wei, and W.-F. Chiou, "The effect of medicinal plants used in Chinese folk medicine on RANTES secretion by virus-infected human epithelial cells," Journal of Ethnopharmacology, vol. 107, no. 2, pp. 205-210, 2006. 


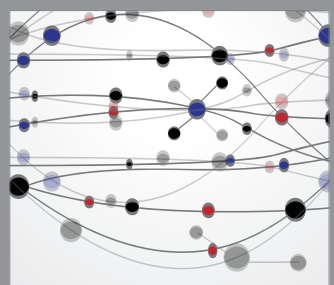

The Scientific World Journal
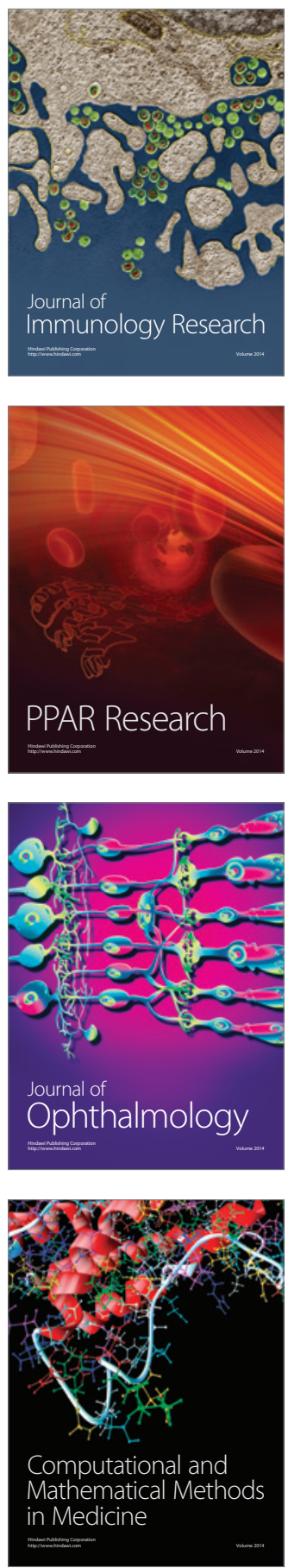

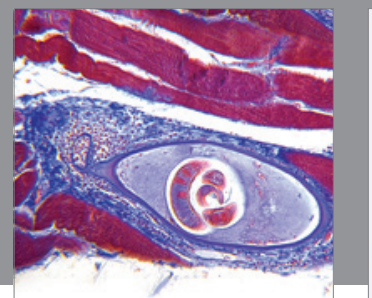

Gastroenterology

Research and Practice
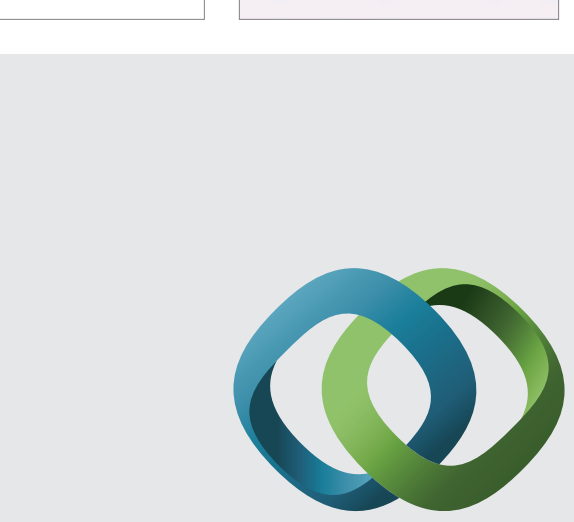

\section{Hindawi}

Submit your manuscripts at

http://www.hindawi.com
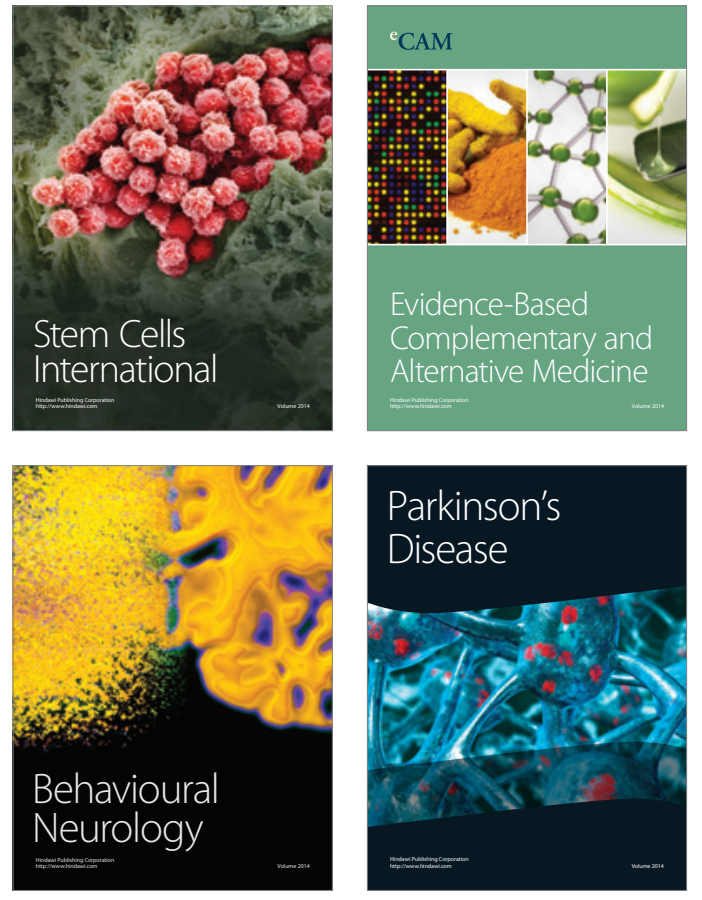
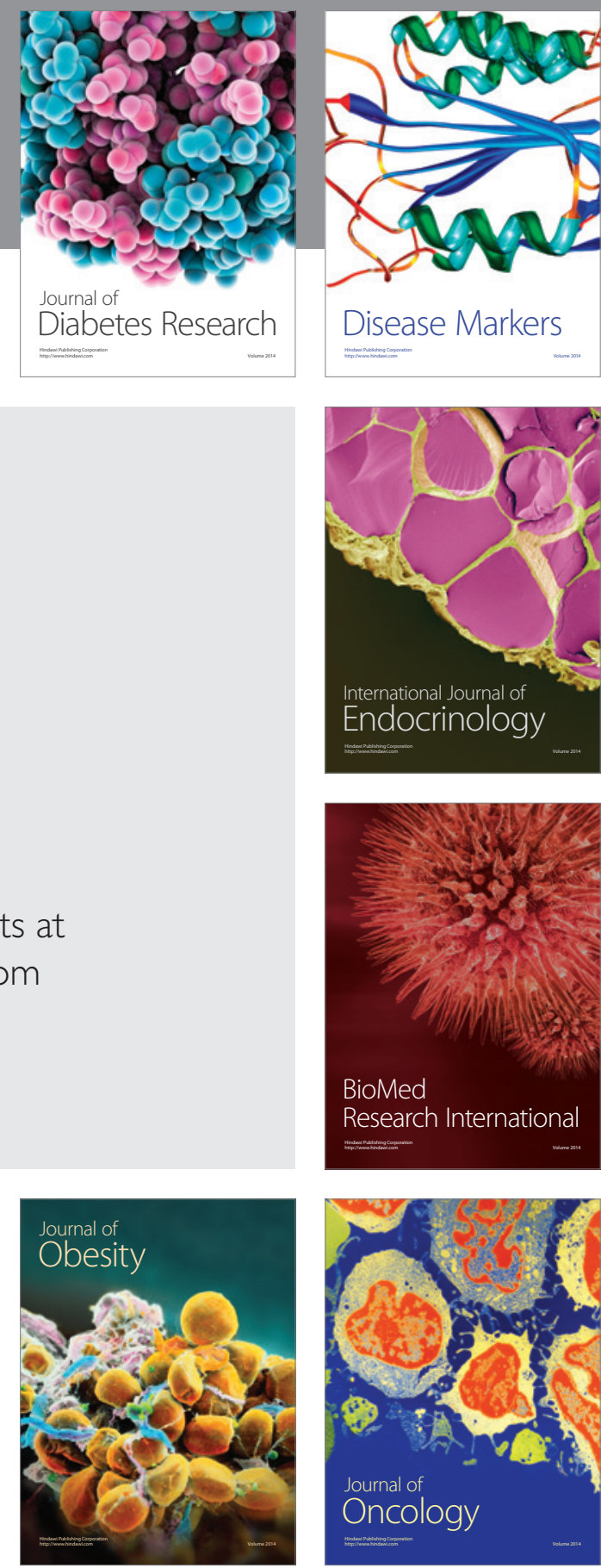

Disease Markers
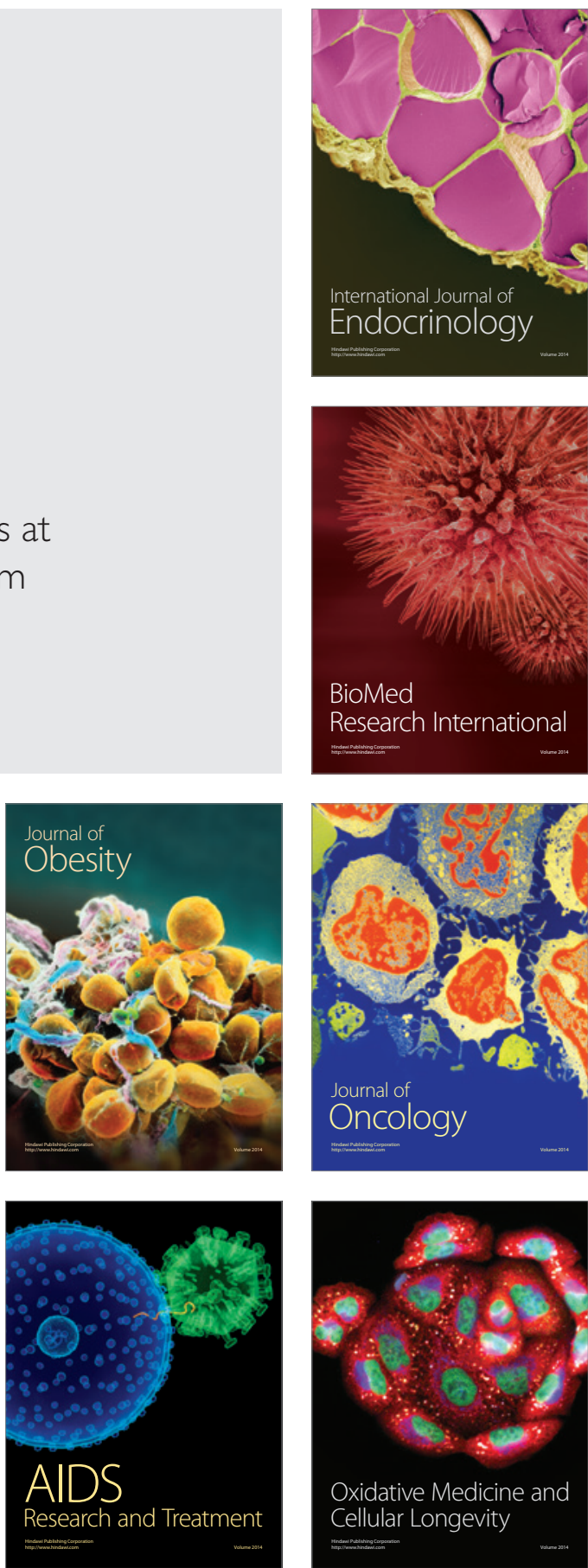